\title{
ALGEBRAIC ORTHOGONALITY AND COMMUTING PROJECTIONS IN OPERATOR ALGEBRAS
}

\author{
ANIL KUMAR KARN
}

\begin{abstract}
We provide an order-theoretic characterization of algebraic orthogonality among positive elements of a general $\mathrm{C}^{*}$-algebra by proving a statement conjectured in [12]. Generalizing this idea, we describe absolutely ordered $p$-normed spaces, for $1 \leq p \leq \infty$ which present a model for "non-commutative vector lattices". Thid notion includes order theoretic orthogonality. We generalize algebraic orthogonality by introducing the notion of absolute compatibility among positive elements in absolute order unit spaces and relate it to symmetrized product in the case of a $\mathrm{C}^{*}$-algebra. In the latter case, whenever one of the elements is a projection, the elements are absolutely compatible if and only if they commute. We develop an order theoretic prototype of the results. For this purpose, we introduce the notion of order projections and extend the results related to projections in a unital $\mathrm{C}^{*}$-algebra to order projections in an absolute order unit space. As an application, we describe spectral decomposition theory for elements of an absolute order unit space.
\end{abstract}

\section{INTRODUCTION}

The order structure of a $\mathrm{C}^{*}$-algebra has been a point of attraction since the inception of the theory. Kakutani's characterization of $C(K)$ spaces ( $K$ a compact, Hausdorff space) as $A M$ - spaces [7] highlighted that the self-adjoint part of a commutative $\mathrm{C}^{*}$-algebra is a Banach lattice (with some additional norm conditions). However, in a non-commutative $\mathrm{C}^{*}$-algebra, join and meet of two general self-adjoint elements may not even exist. Thus it was natural to turn attention towards the non-commutative case. In 1951, Kadison proved that the self-adjoint part of a unital $\mathrm{C}^{*}$-algebra is an order unit space [6]. (However, this was not a characterization as the converse is not true.) Its non-unital version was studied by $\mathrm{Ng}$ [14]. (Also see [3].) The non-self-adjoint version of Kadison's work was introduced by Choi and Effros as matrix order unit spaces [5] whose non-unital version was presented by Karn and Vasudevan [13].

The author carried forward the work further in this direction with an intuition that it may be possible to prove a non-commutative version of Kakutani's theorem. He characterized the (matrix) ordered normed spaces that can be order embedded in $\mathrm{C}^{*}$-algebras [10] and introduced the notion of order smooth $\infty$-normed spaces (order smooth $p$-normed spaces in general, for $1 \leq p \leq \infty$ ) [9]. On such spaces, he studied a notion pf $\infty$-orthogonality ( $p$-orthogonality in general, for

2010 Mathematics Subject Classification. Primary 46B40; Secondary 46L05, 46L30.

Key words and phrases. Absolute $\infty$-orthogonality, absolute order unit space, absolute compatibility, order projection. 
$1 \leq p \leq \infty)$ [11]. In a subsequent paper, he characterized algebraic orthogonality in some classes of $\mathrm{C}^{*}$-algebras (that include commutative $\mathrm{C}^{*}$-algebras as well as von Neumann algebras) in terms of absolute $\infty$-orthogonality (defined for order smooth $\infty$-normed spaces) [12]. In this paper, we extend it to an arbitrary $\mathrm{C}^{*}$-algebra, thus proving Conjecture 4.4 of [12].

Following the above said characterization, the author introduced the notion of an absolute order smooth $p$-normed space $(1 \leq p \leq \infty)$. Thus the examples of an absolute order smooth $\infty$-normed space include the self-adjoint part of an arbitrary $\mathrm{C}^{*}$-algebra. It is important to note that an absolute order smooth p-normed space exhibit a "vector lattice like" structure. More precisely, this structure can be characterized as a vector lattice under an extra condition [12]. In this paper, we shall present a simplified version of this theory to propose a model of a "non-commutative" vector-lattice theory.

Algebraic orthogonal (or equivalently, absolutely orthogonal) pair of positive elements in a $\mathrm{C}^{*}$-algebra are by default commutative. In this paper, we observe that absolutely orthogonal pair of (positive) elements inherit another order theoretic relation which we term as absolute compatibility. We show that for a pair of absolutely compatible positive elements in a $\mathrm{C}^{*}$-algebra, their symmetrized product may be described order theoretically. More precisely, we show that in a $\mathrm{C}^{*}$-algebra $A, a$ and $b$ are mutually absolutely compatible positive elements of $A$ if and only if $\alpha(a \dot{\wedge} b)=a \circ b$ where $\alpha=\max \{\|a\|,\|b\|\}$. (Notions are defined later.) In particular, if one of the elements is a projection, then these elements are absolutely compatible if and only if they commute. These observations indicate that absolute compatibility may be explored as a possible tool to understand commutativity in operator algebras.

In this paper, we develop an order theoretic prototype of these results. For this purpose, we introduce the notion of order projections generalizing projections and extend some of the results related to projections in unital $\mathrm{C}^{*}$-algebras to order projections in absolute order unit spaces. Order projections bear similarity with the notion 'projective units' (defined in order unit spaces) studied in [2] and also with the notion 'projections' (again defined in order unit spaces) studied in [4, 1]. At the end of the paper, as an application, we describe a spectral decomposition theory in an absolute order unit space.

Now we propose the scheme of the paper. In Section 3, we describe absolutely ordered $p$-normed spaces, for $1 \leq p \leq \infty$ which presents a model for "non-commutative" vector lattices and includes order theoretic orthogonality. In section 4, we introduce absolute compatibility between positive elements in an absolute order unit space and relate this notion to symmetrized product in a unital $\mathrm{C}^{*}$-algebra. In Section 5, we introduce order projections as a generalization of projections in operator algebras. We study absolute compatibility of an order projection first, with another order projection in Section 5, and then with general positive elements in Section 6. In Section 7, we discuss a spectral decomposition theory in an absolute order unit space. 


\section{Orthogonality in C*-Algebras}

In [12], we proved that the algebraic orthogonality among positive elements is equivalent to absolutely $\infty$-orthogonality in a von Neumann algebra as well as in a commutative $\mathrm{C}^{*}$-algebra. We begin the paper with proving the result for a general $\mathrm{C}^{*}$-algebra conjectured in [12].

Theorem 2.1. [12, Conjecture 4.4] Let $A$ be a $C^{*}$-algebra. Then for $a, b \in$ $A^{+} \backslash\{0\}$, we have $a b=0$ if and only if \|\|$c\left\|^{-1} c+\right\| d\left\|^{-1} d\right\|=1$ whenever $0<c \leq a$ and $0<d \leq b$ in $A^{+}$.

Let us recall the following result.

Proposition 2.2. [12] Let $A$ be a $C^{*}$-algebra and let $a, b \in A^{+} \backslash\{0\}$. Consider the following statements:

(1) $a b=0$

(2) $0<c \leq a$ and $0<d \leq b$ imply \|\|$c\left\|^{-1} c+\right\| d\left\|^{-1} d\right\|=1$,

(3) $0 \leq c \leq a$ and $c \leq b$ imply $c=0$.

Then (1) implies (2) and (2) implies (3). Further, if $a b=b a$, then (3) implies (1).

Proof. [of Theorem 2.1] ( A. M. Peralta) It suffices to show that $a \perp_{\infty}^{a} b$ implies $a b=0$. Further, without any loss of generality, we may assume that $\|a\|=1=$ $\|b\|$. Let $C^{*}(a)$ be the $\mathrm{C}^{*}$-subalgebra of $A$ generated by $a$. Then $C^{*}(a) \cong C(\sigma(a))$ where $\sigma(a)$ is the spectrum of $a$. Since $\|a\|=1$ we have $\sigma(a) \subset[0,1]$. For each $n \in \mathbb{N}$, we define $a_{n}: \sigma(a) \rightarrow \mathbb{C}$ as follows: For $t \in \sigma(a)$, we set

$$
\begin{aligned}
a_{n}(t) & =t \quad \text { for } t \leq \frac{1}{n} \\
& =\frac{1}{n} \quad \text { for } t \geq \frac{1}{n} .
\end{aligned}
$$

Then $a_{n} \in C(\sigma(a))$ with $a_{n} \leq a$ for each $n$. By functional calculus, $a_{n} \in A^{+}$. Thus by assumption, $a_{n} \perp_{\infty} d$ for any $d \in A^{+}$with $d \leq b$. Also, $\left\|a_{n}\right\|=\frac{1}{n}$ so that $c_{n}:=n a_{n}$ has norm one for each $n$ and that $c_{n} \perp_{\infty} d$ for any $d \in A^{+}$with $d \leq b$. Further we note that $c_{n} \rightarrow[a]$ in $A^{* *}$ in the weak*-topology where $[a]$ is the range projection of a in $A^{* *}$. As the norm in $A^{* *}$ is weak*-lower semi-continuous we have

$$
\|[a]+\| d\left\|^{-1} d\right\| \leq \lim _{w *}\left\|c_{n}+\right\| d\left\|^{-1} d\right\|=1
$$

for $0 \leq d \leq b$. Now as $0 \leq[a] \leq[a]+\|d\|^{-1} d$ we may conclude that $\|[a]+$ $\|d\|^{-1} d \|=1$ we have $[a] \perp_{\infty} d$ whenever $0 \leq d \leq b$. Now by a dual argument, we further get that $[a] \perp_{\infty}[b]$. As $[a]$ and $[b]$ are projections, we have $[a][b]=0$ and consequently, $a b=0$.

\section{ORthogonality in ORDERED VECTOR SPACES}

In this section, we recall few immediate definitions and facts discussed in [9, 11, 12]. we shall present these concepts with a new orientation. This may be seen as a fresh start of the theory of absolutely ordered spaces. The first result is a simpler (and weaker) form of [12, Theorem 4.11]. We include a proof as the 
order structure is proved under weaker assumptions and with a different set of arguments.

Theorem 3.1. Let $V$ be a real vector space. The following sets of conditions on $V$ are equivalent:

(1) There exists a cone $V^{+}$in $V$ and a mapping $|\cdot|: V \rightarrow V^{+}$that satisfies the following conditions:

(a) $|v|=v$ if $v \in V^{+}$.

(b) $|v| \pm v \in V^{+}$.

(c) $|k v|=|k||v|$ for all $v \in V$ and $k \in \mathbb{R}$.

(2) There exists a mapping $\dot{V}: V \times V \rightarrow V$ that satisfies the following conditions:

(a) $v \dot{\vee} v=v$.

(b) $v \dot{\vee} w=w \dot{\vee} v$ for all $v, w \in V$.

(c) $(u \dot{\vee} v)+w=(u+w) \dot{V}(v+w)$ for all $u, v, w \in V$.

(d) $k(v \dot{V} w)=(k v) \dot{V}(k w)$ for all $v, w \in V$ and $k \geq 0$.

(e) If $v \dot{\vee} w=v$, then $(u \dot{\vee} v) \dot{\vee} w=u \dot{\vee}(v \dot{\vee} w)$.

(3) There exists a mapping $\dot{\wedge}: V \times V \rightarrow V$ that satisfies the following conditions:

(a) $v \dot{\wedge} v=v$.

(b) $v \dot{\wedge} w=w \dot{\wedge} v$ for all $v, w \in V$.

(c) $(u \dot{\wedge} v)+w=(u+w) \dot{\wedge}(v+w)$ for all $u, v, w \in V$.

(d) $k(v \dot{\wedge} w)=(k v) \dot{\wedge}(k w)$ for all $v, w \in V$ and $k \geq 0$.

(e) If $v \dot{\wedge} w=v$, then $(u \dot{\wedge} v) \dot{\wedge} w=u \dot{\wedge}(v \dot{\wedge} w)$.

(4) There exists a cone $V^{+}$in $V$ and a binary operation $\perp$ in $V^{+}$that satisfies the following conditions:

(a) $u \perp 0$ for all $u \in V^{+}$.

(b) If $u \perp v$, then $v \perp u$.

(c) If $u \perp v$, then $k u \perp k v$ for all $k \in \mathbb{R}$ with $k>0$.

(d) For each $v \in V$, there exist a unique pair $v_{1}, v_{2} \in V^{+}$such that $v=v_{1}-v_{2}$ with $v_{1} \perp v_{2}$.

Proof. First assume that the set of conditions (1) holds. For $v, w \in V$, we define

$$
v \dot{\vee} w=\frac{1}{2}(v+w+|v-w|)
$$

Then $\dot{V}: V \times V \rightarrow V$ and (2) (a), (b), (c) and (d) follow in a routine way. Further note that $v \dot{\vee} w=v$ if and only if $w \leq v$. Also, $v \leq u \dot{\vee} v$. Now, we show that (2)(e) holds. Let $u, v, w \in V$ with $v \dot{\vee} w=v$. Then $w \leq v \leq u \dot{\vee} v$ so that $(u \dot{\vee} v) \dot{\vee} w=u \dot{\vee} v=u \dot{\vee}(v \dot{\vee} w)$. Thus (1) implies (2).

Next, assume that the set of conditions (2) holds. Set $v \dot{\wedge} w:=v+w-(v \dot{\vee} w)$. Then $\dot{\wedge}: V \times V \rightarrow V$ is a binary mapping such that

$$
v \dot{\wedge} w=v+w-(v \dot{\vee} w)=-\{(-v) \dot{\vee}(-w)\}=\frac{1}{2}(v+w-|v-w|)
$$

for all $v, w \in V$. Now conditions (3)(a) - (3)(e) hold by dual arguments.

Finally, assume the conditions in the set (3). Put

$$
V^{+}=\{v: v \dot{\wedge} 0=0\} .
$$


Let $v \in V^{+}$and $k \geq 0$. Then $v \dot{\wedge} 0=0$ so that using (2)(d), we get $0=$ $k(v \dot{\wedge} 0)=(k v) \dot{\wedge} 0$. Thus $k v \in V^{+}$. Next, note that by using (2)(c) and $(2)(\mathrm{d})$ we can show that $u i n V^{+}$, that is, $u \dot{\wedge} 0=0$ if and only $(-u) \dot{\wedge} 0=-u$ which is equivalent to $u \dot{\wedge}(-u)=-u$. Now, let $v, w \in V^{+}$. Then $-v=v \dot{\wedge}(-v)$ and $-w=w \dot{\wedge}(-w)$ so that $-v-w=(v-w) \dot{\wedge}(-v-w)$ by $(2)$ (c). Similarly, we get $v-w=(v+w) \dot{\wedge}(v-w)$. Thus using (2)(e), we have

$$
\begin{aligned}
(v+w) \dot{\wedge}(-v-w) & =(v+w) \dot{\wedge}\{(v-w) \dot{\wedge}(-v-w)\} \\
& =\{(v+w) \dot{\wedge}(v-w)\} \dot{\wedge}(-v-w) \\
& =(v-w) \dot{\wedge}(-v-w) \\
& =-v-w .
\end{aligned}
$$

Therefore, $V^{+}$is a cone in $V$. Now we define

$$
|v|=-(v \dot{\wedge}(-v))
$$

for all $v \in V$. Note that $v \in V^{+}$if and only if $0=2(v \dot{\wedge} 0)=(2 v) \dot{\wedge} 0=-|v|+v$ so that (1)(a) holds.

To prove $(1)(\mathrm{b})$, first we show that $-(v \dot{\wedge} 0) \in V^{+}$for all $v \in V$. For this, let $v \in V$ and set $w=v \dot{\wedge} 0$. Then

$$
w \dot{\wedge} 0=(v \dot{\wedge} 0) \dot{\wedge} 0=v \dot{\wedge} 0=w
$$

by $(2)(\mathrm{e})$ so that

$$
w \dot{\wedge}(-w)=(2 w) \dot{\wedge} 0-w=2(w \dot{\wedge} 0)-w=w
$$

by $(2)(\mathrm{c})$ and $(2)(\mathrm{d})$. Thus

$$
-w=|w|=|(-w)|
$$

and whence $-(v \dot{\wedge} 0)=-w \in V^{+}$. Now (1)(b) follows from a straight forward observation

$$
|v| \pm v=-2((\mp v) \dot{\wedge} 0)
$$

Now, the proof of $(1)(c)$ directly follows from $(2)(d)$.

Finally, we show the equivalence of (1) and (4). First let the set of conditions (1) hold. For $u, v \in V^{+}$, we define define $u \perp v$ if $|u-v|=u+v$. Then conditions $(4)(\mathrm{a})-(4)(\mathrm{d})$ directly follow from the definition of $\perp$. Conversely, assume that the set of conditions (4) hold. For each $v$, define $|v|:=v_{1}+v_{2}$, using the uniqueness of $(4)(\mathrm{d})$. Then $|\cdot|$ maps $V$ into $V^{+}$. Let $v \in V^{+}$. Since $v \perp 0$ by (4)(a), we have $|v|=v$. Also, by the definition of $|\cdot|$, we further see that $|v| \pm v \in V^{+}$. Now, let $v \in V$. Then by $(4)(\mathrm{d})$, there exists a unique pair $v_{1}, v_{2} \in V^{+}$with $v_{1} \perp v_{2}$ such that $v=v_{1}-v_{2}$. Then $k v_{1} \perp k v_{2}$ for any $k \in \mathbb{R}$ with $k>0$ using condition (4)(c). Now by the definition

$$
|k v|=\left|\left(k v_{1}-k v_{2}\right)\right|=k v_{1}+k v_{2}=k|v| .
$$

Also, if $k \in \mathbb{R}$ with $k<0$, then

$$
|k v|=\left|\left((-k) v_{2}-(-k) v_{1}\right)\right|=(-k) v_{2}+(-k) v_{1}=(-k)|v| .
$$

Thus $|k v|=|k||v|$ for all $k \in \mathbb{R}$. 
Next, we recall Theorem 4.12 of [12].

Theorem 3.2. Let $V$ satisfy the (equivalent sets of) conditions of Theorem 3.1. Then the following statements are equivalent:

(i) $v \dot{\vee} w=\sup \{v, w\}$ for all $v, w \in V$.

(ii) $\dot{\vee}$ is associative in $V$.

(iii) $u \pm v \in V^{+}$implies $|v| \leq u$ for all $u, v \in V$.

(iv) $|v+w| \leq|v|+|w|$ for all $v, w \in V$.

Thus $V$ is a vector lattice if and only if one of the equivalent conditions of this result (in addition to the equivalent set of conditions of Theorem 3.1) holds in $V$.

Remark 3.3. In addition to the (equivalent sets of) conditions of Theorem 3.1, there are some other properties which hold both in a vector lattice as well as in a $\mathrm{C}^{*}$-algebra.

Let $V$ be a vector lattice.

(1) If $u, v, w \in V$ with $u \wedge v=0$ and $u \wedge w=0$, then $u \wedge(v+w)=0$. To see this, first note that $x \wedge y \leq x$ for any $x, y \in V$ so that $u, v, w \in V^{+}$. Now, as $u \wedge v=0$, we get $(u+w) \wedge(v+w)=w$. Thus

$$
0=u \wedge w=u \wedge(u+w) \wedge(v+w)=u \wedge(v+w)
$$

for $u \leq u+w$.

(2) Let $u, v \in V$ with $u \wedge v=0$. If $0 \leq w \leq v$, then $u \wedge w=0$. In fact, $w \wedge v=w$ so that

$$
u \wedge w=u \wedge v \wedge w=0 \wedge w=0 .
$$

In particular, if $u, v, w \in V^{+}$with $u \wedge(v+w)=0$, then $u \wedge v$ and $u \wedge w=0$.

(3) If $u, v, w \in V$ with $u \wedge v=0$ and $u \wedge w=0$, then $u \wedge|v-w|=0$. This follows from (1) and (2) as $|v-w| \leq v+w$.

Next, let $A$ be a $C^{*}$-algebra.

(4) For $a \in A$ we define $|a|:=\left(a^{*} a\right)^{\frac{1}{2}}$. Then $\left(A_{s a}, A^{+},|\cdot|\right)$ satisfies the set of conditions (1) of Theorem 3.1. Also, (1), (2) and (3) hold in $A_{s a}$ as well when we replace $\wedge$ by $\dot{\wedge}$. In fact, we have $a \dot{\wedge} b=0$ if and only if $a, b \in A^{+}$and $a b=0$. To see this, first we note that $a \dot{\wedge} b=0$ if and only if $|a-b|=a+b$. We show that $|a-b|=a+b$ if and only if $a, b \in A^{+}$ with $a b=0$. First, let $|a-b|=a+b$. Then

$$
a^{2}-a b-b a+b^{2}=|a-b|^{2}=(a+b)^{2}=a^{2}+a b+b a+b^{2}
$$

so that $a b+b a=0$. Also, in this case,

$$
(a+b) \pm(a-b)=|a-b| \pm(a-b) \in A^{+}
$$

so that $a, b \in A^{+}$. Thus $-a^{2} b=a b a \in A^{+}$and consequently, $a^{2} b=b a^{2}$. Now, by the functional calculus in $A$, we can conclude that $a b=b a$. Consequently, $a b=0$. Conversely, if $a, b \in A^{+}$with $a b=0$, then $a b=b a$ and using the (commutative) $\mathrm{C}^{*}$-algebra generated by $a$ and $b$, we can show that $|a-b|=a+b$ so that $a \dot{\wedge} b=0$. 
(5) We have not been able to prove whether (1), (2) and (3) (with $\wedge$ replaced by $\dot{\lambda}$ ) will follow from the equivalent conditions of Theorem 3.1 or not. Also, we find these properties useful for the develop the theory. Thus we shall include them in the definition of an absolutely ordered space.

(6) Let $V$ satisfy the equivalent conditions of Theorem 3.1 and let $u, v \in V$. Then $u \dot{\wedge} v=0$ if and only if $u, v \in V^{+}$and $|u-v|=u+v$ (or equivalently, $u \perp v)$. Further, $|2 u-v|=v$ if and only if $0 \leq u \leq v$ and $u \perp(v-u)$.

Definition 3.4. Let $\left(V, V^{+}\right)$be a real ordered vector space and let $|\cdot|: V \rightarrow V^{+}$ be a mapping satisfying the following conditions:

(1) $|v|=v$ if $v \in V^{+}$;

(2) $|v| \pm v \in V^{+}$

(3) $|k v|=|k||v|$ for all $v \in V$ and $k \in \mathbb{R}$;

(4) If $|u-v|=u+v$ and $0 \leq w \leq v$, then $|u-w|=u+w$; and

(5) If $|u-v|=u+v$ and $|u-w|=u+w$, then $|u-(v \pm w)|=u+|v \pm w|$.

Then $\left(V, V^{+},|\cdot|\right)$ will be called an absolutely ordered space.

\subsection{Norms on absolutely ordered vector spaces.}

Definition 3.5. Let $\left(V, V^{+}\right)$be a real ordered vector space such that $V^{+}$is proper and generating and let $\|\cdot\|$ be a norm on $V$ such that $V^{+}$is $\|\cdot\|$-closed. Let $1 \leq p \leq \infty$. For $u, v \in V^{+}$, we say that $u$ is $p$-orthogonal to $v$, (we write, $u \perp_{p} v$ ), if

$$
\begin{aligned}
\|\alpha u+\beta v\| & =\left(\|\alpha u\|^{p}+\|\beta v\|^{p}\right)^{\frac{1}{p}} \quad(1 \leq p<\infty) \\
& =\max (\|\alpha u\|,\|\beta v\|) \quad(p=\infty)
\end{aligned}
$$

Further, we say that $u$ is absolutely $p$-orthogonal to $v$, (we write, $u \perp_{p}^{a} v$ ), if $u_{1} \perp_{p} v_{1}$ whenever $0 \leq u_{1} \leq u$ and $0 \leq v_{1} \leq v$.

The following observation describes the importance of $\perp_{p}$.

Proposition 3.6. Let $\left(V, V^{+},|\cdot|\right)$ be an absolutely ordered vector space and assume that $\|\cdot\|$ is a norm on $V$ such that $V^{+}$is $\|\cdot\|$-closed. Then for $1 \leq p \leq \infty$, the following conditions are equivalent:

(A) For each $v \in V$, we have

$$
\begin{aligned}
\||v|\|=\|v\| & =\left(\left\|v^{+}\right\|^{p}+\left\|v^{-}\right\|^{p}\right)^{\frac{1}{p}} \quad(1 \leq p<\infty) \\
& =\max \left(\left\|v^{+}\right\|,\left\|v^{-}\right\|\right) \quad(p=\infty) ;
\end{aligned}
$$

(B) For $u, v \in V^{+}$, we have $u \perp_{p}^{a} v$ whenever $u \perp v$;

(C) For $u, v \in V^{+}$, we have $u \perp_{p} v$ whenever $u \perp v$.

If $\|\cdot\|$ is an order unit norm determined by the order unit $e$, then the above conditions (with $p=\infty$ ) are also equivalent to:

(D) For each $v \in V$ with $\pm v \leq e$, we have $|v| \leq e$.

Proof. First, assume that (A) holds. Let $u, v \in V^{+}$with $u \perp v$ and suppose that $0 \leq u_{1} \leq u$ and $0 \leq v_{1} \leq v$. Then for $k, l \in \mathbb{R}$ with $k, l>0$, we have $k u_{1} \perp l v_{1}$. 
If we set $w=k u_{1}-l v_{1}$, then $|w|=k u_{1}+l v_{1}, w^{+}=k u_{1}$ and $w^{-}=l v_{1}$. Thus by (A), we have

$$
\begin{aligned}
\left\|k u_{1} \pm l v_{1}\right\| & =\left(\left\|k u_{1}\right\|^{p}+\left\|l v_{1}\right\|^{p}\right)^{\frac{1}{p}} \quad(1 \leq p<\infty) \\
& =\max \left(\left\|k u_{1}\right\|,\left\|l v_{1}\right\|\right) \quad(p=\infty)
\end{aligned}
$$

so that $u \perp_{p}^{a} v$. Thus (A) implies (B). Now that (B) implies (C) is trivial.

Next, assume that $(\mathrm{C})$ holds. Let $v \in V$. Then $v^{+} \perp v^{-}$. Thus by assumption, $v^{+} \perp_{p} v^{-}$so that

$$
\begin{aligned}
\left\|v^{+} \pm v^{-}\right\| & =\left(\left\|v^{+}\right\|^{p}+\left\|v^{-}\right\|^{p}\right)^{\frac{1}{p}} \quad(1 \leq p<\infty) \\
& =\max \left(\left\|v^{+}\right\|,\left\|v^{-}\right\|\right) \quad(p=\infty) .
\end{aligned}
$$

Since $v=v^{+}-v^{-}$and $|v|=v^{+}+v^{-}$, we see that (A) holds.

Now, assume that $\|\cdot\|$ is an order unit norm determined by the order unit $e$. First let (D) hold. Let $u, v \in V^{+}$with $u \perp v$. We show that $u \perp_{\infty} v$. Without any loss of generality, we may assume that $\|u\|=1=\|v\|$. Set $w=u-v$. As $-v \leq w \leq u$, we have $\|w\| \leq \max \{\|u\|,\|v\|\}=1$ so that $\pm w \leq e$. Now, by assumption, $u+v=|w| \leq e$. Thus $1=\|u\| \leq\|u+v\| \leq 1$ so that $u \perp_{\infty} v$.

Finally, assume that (A) holds. Let $v \in V$ with $\pm v \leq e$. Then $\|v\| \leq 1$. Now, by assumption, $\||v|\| \leq 1$ so that $|v| \leq e$. This completes the proof.

Definition 3.7. Let $\left(V, V^{+},|\cdot|\right)$ be an absolutely ordered vector space and assume that $\|\cdot\|$ is a norm on $V$ such that $V^{+}$is $\|\cdot\|$-closed. For $1 \leq p \leq \infty$, we say that $\left(V, V^{+},|\cdot|,\|\cdot\|\right)$ is an absolutely order smooth p-normed space, if it satisfies the following conditions.

(O.p.1): For $u \leq v \leq w$ we have

$$
\begin{aligned}
\|v\| & \leq\left(\|u\|^{p}+\|w\|^{p}\right)^{\frac{1}{p}} \quad(1 \leq p<\infty) \\
& \leq \max (\|u\|,\|w\|) \quad(p=\infty) ; \quad \text { and }
\end{aligned}
$$

$\left(O . \perp_{p} .1\right)$ : For $u, v \in V^{+}$with $u \perp v$, we have $u \perp_{p}^{a} v$.

$\left(O . \perp_{p} .2\right)$ : For $u, v \in V^{+}$with $u \perp_{p}^{a} v$, we have $u \perp v$.

If in addition, (for $p=\infty$, ) $\|\cdot\|$ is an order unit norm on $V$ determined by an order unit $e$, we say that $(V, e)$ is an absolute order unit space.

Remark 3.8.

(1) The self-adjoint part of every $\mathrm{C}^{*}$-algebra is an absolutely order smooth $\infty$-normed space.

(2) The self-adjoint part of the dual of any $\mathrm{C}^{*}$-algebra is an absolutely order smooth 1-normed space.

(3) In general, $T_{p}(H)_{s a}$, the self-adjoint part of the space of trace class operators on a complex Hilbert space $H$ is an absolutely order smooth $p$-normed space.

(4) Let $\left(V, V^{+},|\cdot|,\|\cdot\|\right)$ is an absolutely order smooth $p$-normed space. Assume that $u, v, w \in V^{+}$with $u \perp v$ and $u \perp w$. Then for $\alpha, \beta>0$, we have $u \perp \alpha v$ and $u \perp \beta w$ and consequently, $u \perp|\alpha v+\beta w|$. Thus $u \perp_{p}(\alpha v+\beta w)$ for all $\alpha, \beta \in \mathbb{R}$. 
(5) Recall that an order smooth $p$-normed space is a real ordered vector space $\left(V, V^{+}\right)$in which $V^{+}$is proper and generating together with a norm $\|\cdot\|$ for which $V^{+}$is closed such that the space satisfies the conditions (O.p.1) and

(O.p.2): For each $v \in V$ and $\epsilon>0$, there exist $v_{1}, v_{2} \in V^{+}$such that $v=v_{1}-v_{2}$ and

$$
\begin{aligned}
\|v\|+\epsilon & >\left(\left\|v_{1}\right\|^{p}+\left\|v_{2}\right\|^{p}\right)^{\frac{1}{p}} \quad(1 \leq p<\infty) \\
& >\max \left(\left\|v_{1}\right\|,\left\|v_{2}\right\|\right) \quad(p=\infty) .
\end{aligned}
$$

Thus an absolutely order smmoth $p$-normed space is an order smooth $p$ normed space satisfying

(OS.p.2): For each $v \in V$, there exist $v_{1}, v_{2} \in V^{+}$such that $v=v_{1}-v_{2}$ and

$$
\begin{aligned}
\|v\| & =\left(\left\|v_{1}\right\|^{p}+\left\|v_{2}\right\|^{p}\right)^{\frac{1}{p}} \quad(1 \leq p<\infty) \\
& =\max \left(\left\|v_{1}\right\|,\left\|v_{2}\right\|\right) \quad(p=\infty) .
\end{aligned}
$$

(6) Let $\left(V, V^{+}, e\right)$ be an order unit space. Then, with the order unit norm $\|\cdot\|_{e}$ on $V,\left(V, V^{+},\|\cdot\|_{e}\right)$ is an order smooth $\infty$-normed space satisfying $(O S . \infty .2)$.

\section{Absolute orthogonality And Symmetrized ProduCt}

By the definition, algebraic orthogonal pair of positive elements in a $\mathrm{C}^{*}$-algebra commute. In this section, we introduce an order theoretic notion, defined forpositive elements, which nearly imitates commutativity when considered in a $\mathrm{C}^{*}$-algebra.

Proposition 4.1. Let $(V, e)$ be an absolute order unit space and assume that $u, v \in[0, e]$. Then $u \perp v$ if and only if $u+v \leq e$ and $|u-v|+|u+v-e|=e$.

Proof. Let $u \perp v$. Then $|u-v|=u+v$ and $\|u+v\|=\max \{\|u\|, \mid v \|\} \leq 1$. Thus $u+v \in[0, e]$ so that

$$
|u+v-e|=e-(u+v)=e-|u-v| .
$$

Now, it follows that $|u-v|+|u+v-e|=e$. Conversely, assume that $u+v \leq e$ and that $|u-v|+|u+v-e|=e$. Then

$$
e-|u-v|=|u+v-e|=e-(u+v)
$$

so that $|u-v|=u+v$. In other words, $u \perp v$.

Proposition 4.2. Let $(V, e)$ be an absolute order unit space. If $|u-v|+|u+v-e|=$ $e$, then $u, v \in[0, e]$.

Proof. Assume that $\alpha=1$. Then $|u-v|+|u+v-e|=e$. Thus we have

$$
\begin{aligned}
0 & \leq|u-v| \pm(u-v)+|u+v-e| \pm(u+v-e) \\
& =e \pm(u-v) \pm(u+v-e) .
\end{aligned}
$$

Thus

$$
e+(u-v)+(u+v-e) \geq 0
$$




$$
\begin{gathered}
e-(u-v)+(u+v-e) \geq 0 \\
e+(u-v)-(u+v-e) \geq 0
\end{gathered}
$$

and

$$
e-(u-v)-(u+v-e) \geq 0 .
$$

Now, it follows that $u, v \in[0, e]$.

Definition 4.3. Let $(V, e)$ be an absolute order unit space. Then $u, v \in[0, e]$ are said to be absolutely comparable, if $|u-v|+|u+v-e|=e$.

Proposition 4.4. Let $(V, e)$ be an absolute order unit space. Then for $u, v \in[0, e]$ the following statements are equivalent:

(1) $u$ is absolutely compatible with $v$;

(2) $u \dot{\wedge} v+u \dot{\wedge}(e-v)=u$;

(3) $u \dot{\wedge} v+(e-u) \dot{\wedge} v=v$

(4) $(e-u) \dot{\wedge} v+(e-u) \dot{\wedge}(e-v)=e-u$;

(5) $u \dot{\wedge}(e-v)+(e-u) \dot{\wedge}(e-v)=e-v$;

(6) $u \dot{\wedge} v+u \dot{\wedge}(e-v)+(e-u) \dot{\wedge} v+(e-u) \dot{\wedge}(e-v)=e$.

Proof. Using symmetry in the condition for absolute compatibility, we may conclude that $u$ is absolutely compatible with $v$ if and only if $\{u, e-u\}$ is absolutely compatible with $\{v, e-v\}$. Next, as

$$
\begin{aligned}
u \dot{\wedge} v+u \dot{\wedge}(e-v) & =\frac{1}{2}\{u+v-|u-v|+u+e-v-|u-e+v|\} \\
& =u+\frac{1}{2}\{e-(|u-v|+|u+v-e|)\}
\end{aligned}
$$

we conclude that $u$ and $v$ are absolutely compatible with respect to $p$ if and only if $u \dot{\wedge} v+u \dot{\wedge}(e-v)=u$. Now combining the two observations, the proofs follow in a routine way.

Proposition 4.5. Let $(V, e)$ be an absolute order unit space. Then for $u, v \in[0, e]$ the following statements are equivalent:

(1) $u$ is absolutely compatible with $v$;

(2) $u \dot{\wedge} v,(e-u) \dot{\wedge}(e-v) \in V^{+}$with $u \dot{\wedge} v \perp(e-u) \dot{\wedge}(e-v)$;

(3) $u \dot{\wedge}(e-v),(e-u) \dot{\wedge} v \in V^{+}$with $u \dot{\wedge}(e-v) \perp(e-u) \dot{\wedge} v$.

Proof. First, let us assume that $u$ is absolutely compatible with $v$ so that $\mid u-$ $v|+| u+v-e \mid=e$. Then

$$
\begin{aligned}
u \dot{\wedge} v & =\frac{1}{2}\{u+v-|u-v|\} \\
& =\frac{1}{2}\{u+v-e+|u+v-e|\} \\
& =(u+v-e)^{+} .
\end{aligned}
$$

In a similar manner, we can also show that

$$
\begin{aligned}
& u \dot{\wedge}(e-v)=(u-v)^{+} \\
& (e-u) \dot{\wedge} v=(u-v)^{-}
\end{aligned}
$$


and

$$
(e-u) \dot{\wedge}(e-v)=(u+v-e)^{-} .
$$

Thus $u \dot{\wedge} v, u \dot{\wedge}(e-v),(e-u) \dot{\wedge} v,(e-u) \dot{\wedge}(e-v) \in V^{+}$with

$$
u \dot{\wedge} v \perp(e-u) \dot{\wedge}(e-v)
$$

and

$$
u \dot{\wedge}(e-v) \perp(e-u) \dot{\wedge} v .
$$

Thus (1) implies (2) and (3).

Conversely, let $u \dot{\wedge} v,(e-u) \dot{\wedge}(e-v) \in V^{+}$with $u \dot{\wedge} v \perp(e-u) \dot{\wedge}(e-v)$. Then by the definition, we have

$$
u \dot{\wedge} v+(e-u) \dot{\wedge}(e-v)=e-|u-v|
$$

and

$$
u \dot{\wedge} v-(e-u) \dot{\wedge}(e-v)=u+v-e .
$$

Now, $u \dot{\wedge} v \perp(e-u) \dot{\wedge}(e-v)$ implies that $|u+v-e|=e-|u-v|$, that is, $u$ is absolutely compatible with $v$.

Dually, $u$ is absolutely compatible with $v$ if and only if $u \dot{\wedge}(e-v),(e-u) \dot{\wedge} v \in V^{+}$ with $u \dot{\wedge}(e-v) \perp(e-u) \dot{\wedge} v$.

Remark 4.6. Let $u, v \in[0, e]$. Since $(e-u) \dot{\wedge}(e-v)=e-(u \dot{\vee} v)$, it follows from Proposition 4.5 that $u$ is absolutely compatible with $v$ if and only if $u \dot{\wedge} v, u \dot{\vee} v \in$ $[0, e]$ with $u \dot{\wedge} v \perp(e-u \dot{\vee} v)$. Thus by Proposition 4.1, $u$ is absolutely compatible with $v$ if and only if $u \dot{\wedge} v$ is absolutely compatible with $u \dot{\vee} v$. In particular, $u$ is absolutely compatible to itself if and only if $u \perp(e-u)$.

Let $A$ be a $C^{*}$-algebra. For $a, b \in A$ we shall write, $a \circ b:=\frac{1}{2}(a b+b a)$.

Proposition 4.7. Let $A$ be a unital $C^{*}$-algebra. Then for $a, b \in A_{\text {sa }}$, we have $|a-b|+|a+b-1|=1$ if and only if $a, b \in[0,1]$ with $a \circ b=a \dot{\wedge} b$.

Proof. First let $|a-b|+|a+b-1|=1$. Note that $|x| \pm x \in A^{+}$for all $x \in A_{s a}$. Thus $1 \pm(a-b) \pm(a+b-1) \in A^{+}$so that $a, b \in[0,1]$. Now

$$
|a+b-1|^{2}=a^{2}+b^{2}+1+a b+b a-2 a-2 b
$$

and

$$
(1-|a-b|)^{2}=1+a^{2}+b^{2}-a b-b a-2|a-b| .
$$

Also, by assumption, we have $|a+b-1|=1-|a-b|$ so that

$$
\begin{aligned}
a^{2}+b^{2}+1+a b+b a-2 a-2 b & =|a+b-1|^{2} \\
& =(1-|a-b|)^{2} \\
& =1+a^{2}+b^{2}-a b-b a-2|a-b|
\end{aligned}
$$

Thus $2(a b+b a)=2(a+b-|a-b|)$ so that $a \circ b=a \dot{\wedge} b$.

Conversely, let $a, b \in[0,1]$ with $a \circ b=a \dot{\wedge} b$. Expanding $a \circ b=a \dot{\wedge} b$ as above, we can show that $|a+b-1|^{2}=(1-|a-b|)^{2}$. Now, as $a, b \in[0,1]$, we have

$$
-1 \leq-b \leq a-b \leq a \leq 1
$$

so that $|a-b| \leq 1$. Thus $|a+b-1|=1-|a-b|$. 
Corollary 4.8. Let $A$ be a unital $C^{*}$-algebra and $a, b \in A^{+}$. Set $\alpha=\max \{\|a\|,\|b\|\}$. Then $a$ is absolutely comparable with $b$ if and only if $a \circ b=\alpha(a \dot{\wedge} b)$.

For a projection, Proposition 4.7 takes the following form.

Proposition 4.9. Let $A$ be a unital $C^{*}$-algebra. Then for $0 \leq a \leq 1$ in $A$ and for a projection $p$ in $A$, the following statements are equivalent:

(1) a is absolutely comparable with $p$;

(2) $a \dot{\wedge} p=a p$;

(3) $a p=p a$.

In this case, $\inf \{a, p\}$ exists (in $A^{+}$) and is equal to $a \dot{\wedge} p$.

Proof. (1) $\Longrightarrow(2)$ : First, assume that $|a-p|+|a+p-1|=1$. Let $a-p=x_{1}-x_{2}$ and $a+p-1=y_{1}-y_{2}$ such that $x_{1}, x_{2}, y_{1}, y_{2} \in A^{+}$with $x_{1} x_{2}=0$ and $y_{1} y_{2}=0$. Then $|a-p|=x_{1}+x_{2}$ and $|a+p-1|=y_{1}+y_{2}$. Thus $x_{1}+x_{2}+y_{1}+y_{2}=1$ and $x_{1}-x_{2}+y_{1}-y_{2}=2 a-1$ so that $a=x_{1}+y_{1}$ and $p=x_{2}+y_{1}$ and consequently, $1-p=x_{1}+y_{2}$. As $p$ and $1-p$ are projections with $p(1-p)=0$, it follows that $x_{1} y_{1}=0$ and $x_{2} y_{2}=0$. In particular, $x_{1} p=0$ so that $a p=y_{1}$. Thus

$$
a \dot{\wedge} p=\frac{1}{2}\{a+p-|a-p|\}=y_{1}=a p .
$$

As $a \dot{\wedge} p$ is self-adjoint, (2) $\Longrightarrow(3)$ is evident.

$(3) \Longrightarrow(1)$ : Next, assume that $a p=p a$. Then $a p=p a p$ and $a(1-p)=$ $(1-p) a(1-p)$ so that $a=p a p+(1-p) a(1-p)$. As $0 \leq a \leq 1$, we see that $0 \leq p a p \leq p$ and $0 \leq(1-p) a(1-p) \leq(1-p)$. Thus

$$
\begin{aligned}
|a-p| & =|(1-p) a(1-p)-(p-p a p)| \\
& =(1-p) a(1-p)+(p-p a p)
\end{aligned}
$$

and

$$
\begin{aligned}
|a+p-1| & =|((1-p)-(1-p) a(1-p))-p a p| \\
& =((1-p)-(1-p) a(1-p))+\text { pap } .
\end{aligned}
$$

Adding them, we get $|a-p|+|a+p-1|=1$.

Finally, assume that $a p=p a$. Then $a p=a^{\frac{1}{2}} p a^{\frac{1}{2}} \leq a$ and $a p=p a p \leq p$. As $a, p \in A^{+}$and $a p=p a$, we have $a p \in A^{+}$. Next, let $x \in A^{+}$be such that $x \leq a$ and $x \leq p$. As $p$ is a projection, we get $x p=p x=x$. Thus

$$
x=p x p \leq p a p=a p
$$

so that $a \dot{\wedge} p=a p=\inf \{a, p\}$.

Corollary 4.10. Let $A$ be a unital $C^{*}$-algebra. Then for $a \in A^{+}$and for $a$ projection $p$ in $A$, the following statements are equivalent:

(1) a is absolutely comparable with $p$;

(2) $a \dot{\wedge} p=a p$;

(3) $a p=p a$.

Commuting projections yield the following refinement of Proposition 4.9. 
Proposition 4.11. Let $A$ be a unital $C^{*}$-algebra and let $\mathcal{P}(A)$ denote the set of projections in $A$. Then for $p, q \in \mathcal{P}(A)$, we have $p q=q p$ if and only if $p \dot{\wedge} q \in \mathcal{P}(A)$. In this case, $p \dot{\wedge} q=p q=\inf _{\mathcal{P}(A)}\{p, q\}$.

Proof. First let $p q=q p:=r$. Then $r=\inf _{\mathcal{P}(A)}\{p, q\}$. We show that $r=p \dot{\wedge} q$. Since $p-r, q-r \in \mathcal{P}(A)$ with $(p-r)(q-r)=0$, we get

$$
|p-q|=|(p-r)-(q-r)|=(p-r)+(q-r) .
$$

Thus $p \dot{\wedge} q=\frac{1}{2}\{p+q-|p-q|\}=r$.

Conversely, assume that $p \dot{\wedge} q \in \mathcal{P}(A)$. Since $p \dot{\wedge} q \leq p$ and $p \dot{\wedge} q \leq q$, we get $p(p \dot{\wedge} q)=(p \dot{\wedge} q) p=p \dot{\wedge} q$ and $q(p \dot{\wedge} q)=(p \dot{\wedge} q) q=p \dot{\wedge} q$. Also, then

$$
|(p-p \dot{\wedge} q)-(q-p \dot{\wedge} q)|=|p-q|=p+q-2(p \dot{\wedge} q),
$$

so that $(p-p \dot{\wedge} q)(q-p \dot{\wedge} q)=0=(q-p \dot{\wedge} q)(p-p \dot{\wedge} q)$. Thus $p q=q p=p \dot{\wedge} q$.

\section{Commuting Projections}

Now we shall present order theoretic replicas of Propositions 4.9 and 4.11.

Definition 5.1. Let $V$ be an ordered vector space. For $u \in V^{+}$we set

$$
V_{u}:=\left\{v \in V: k u \pm v \in V^{+} \text {for some } k>0\right\} .
$$

If $(V, e)$ is an order unit space, then $u \in V^{+}$is said to have the order unit property in $V$, if for any $v \in V_{u}$ we have, $\pm v \leq\|v\| u$. In this case, $\left(V_{u}, u\right)$ is also an order unit space and $\|v\|_{u}=\|v\|_{e}$ for each $v \in V_{u}$.

If $(V, e)$ is an absolute order unit space, then $u \in V^{+}$is said to have the absolute order unit property in $V$, if for any $v \in V_{u}$ we have, $|v| \leq\|v\| u$. In this case, $\left(V_{u}, u\right)$ is also an absolute order unit space and $\|v\|_{u}=\|v\|_{e}$ for each $v \in V_{u}$.

Definition 5.2. Let $(V, e,|\cdot|)$ is an absolute order unit space. Consider the set

$$
O P(V)=\{p \in[0,1]: p \perp(e-p)\} .
$$

Note that $0, e \in O P(V)$ and that $e-p \in O P(V)$ if $p \in O P(V)$. We shall write $p^{\prime}$ for $e-p \in O P(V)$. Elements of $O P(V)$ will be called order projections for the following reason.

Theorem 5.3. Let $A$ be a unital $C^{*}$-algebra. For $a \in[0,1]$, these statements are equivalent:

(1) a is a projection in $A$;

(2) a is an extreme point of $[0,1]$;

(3) a has the order unit property in $A$;

(4) $[0, a] \cap[0,1-a]=\{0\}$;

(5) $a \perp(1-a)$.

Proof. Whereas the equivalence of (1) and (2) is a classical result of $\mathrm{C}^{*}$-algebra theory, the equivalence of (1) and (3) was observed in [8, Corollary 3.2 and Theorem 3.3]. Further, as $a$ and $1-a$ commute, the equivalence of (4) and (5) follows from [12, Proposition 4.1]. 
(1) implies (4): Assume that $a^{2}=a$ and let $x \in[0, a] \cap[0,1-a]$. Then $0 \leq x \leq a$ and $0 \leq x \leq 1-a$. Thus

$$
0 \leq(1-a) x(1-a) \leq(1-a) a(1-a)=0
$$

so that $(1-a) x(1-a)=0$ and consequently, $x=a x=x a=a x a$. Now as $0 \leq x \leq 1-a$, as above we get $a x a=0$ so that $x=0$.

(4) implies (1): Finally, assume that $[0, a] \cap[0,1-a]=\{0\}$. As $0 \leq a \leq 1$, we have $0 \leq a^{2} \leq a$ and consequently, $0 \leq a-a^{2} \leq a$. Also,

$$
a-a^{2}=(1-a)^{\frac{1}{2}} a(1-a)^{\frac{1}{2}} \leq(1-a)
$$

so that by assumption, $a-a^{2}=0$. Thus $a$ is a projection.

Remark 5.4. Note that $a$ is a projection if and only if $1-a$ is also a projection. Thus we can replace $a$ by $1-a$ in (1), (2) and (3) of Theorem 5.3.

Throughout the section, $V$ will be an absolute order unit space and $O P(V)$ will denote the set of all order projections in $V$.

Proposition 5.5. For $p, q \in O P(V)$, the following statements are equivalent:

(1) $p+q \leq e$;

(2) $p \perp q$;

(3) $p+q \in O P(V)$, and

(4) $p \perp_{\infty} q$.

Proof. (1) implies (2): Let $p+q \leq e$. Then $0 \leq p \leq e-q$ so that $p \perp q$, by the definition.

(2) implies (4): By Proposition 3.6.

(4) imlies (1): Let $p \perp_{\infty} q$. Then $\|p+q\|=\max \{\|p\|, \mid q \|\} \leq 1$ so that $p+q \leq e$.

(1) implies (3): Let $p+q \leq e$ so that $p \leq e-q$. As $p, q \in O P(V)$, we have $p \perp e-p$ and $q \perp e-q$. Now, $0 \leq e-(p+q) \leq e-p$ and $e-(p+q) \leq e-q$. Thus $e-(p+q) \perp p$ and $e-(p+q) \perp q$. It follows from the additivity of $\perp$ that $e-(p+q) \perp p+q$ so that $p+q \in O P(V)$. Finally, (3) implies (1) by the definition of $O P(V)$.

Proposition 5.6. Let $u, v \in[0, e]$. If $u+v \in O P(V)$ with $u \perp v$, then $u, v \in$ $O P(V)$.

Proof. Set $p=u+v$ so that $u \perp(p-u)$. Since $p \in O P(V)$, we have $p \perp(e-p)$. Since $u \leq p$, we get $u \perp(e-p)$. Now by the additivity of $\perp$, we get $u \perp(e-u)$ so that $u \in O P(V)$. Similarly, we can show that $v \in O P(V)$.

Corollary 5.7. Let $p, q \in O P(V)$ such that $p \leq q$. Then $q-p \in O P(V)$.

Proof. Set $q-p=r$ so that $r \in[0, e]$. Since $0 \leq r \leq e-p$ and $p \perp(e-p)$, we have $p \perp r$. Thus by Proposition 5.6, $r \in O P(V)$.

Remark 5.8. Let $p, q \in O P(V)$ such that $p \leq q$. Then $p \perp(q-p)$.

Proposition 5.9. Let $u, v \in V$. Then

(1) $u \dot{\vee} v+u \dot{\wedge} v=u+v$;

(2) $u \dot{\vee} v-u \dot{\wedge} v=|u-v|$; 
(3) $u-u \dot{\wedge} v, v-u \dot{\wedge} v \in V^{+}$; and

(4) $(u-u \dot{\wedge} v) \perp(v-u \dot{\wedge} v)$.

Proof. The statements (1), (2) and (3) follow from the definitions of $\dot{\lambda}$ and $\dot{\boldsymbol{V}}$; and (4) follows from the fact that

$$
(u-u \dot{\wedge} v) \dot{\wedge}(v-u \dot{\wedge} v)=u \dot{\wedge} v-u \dot{\wedge} v=0 .
$$

Theorem 5.10. Let $V$ be an absolute order unit space and let $p, q \in O P(V)$. Then the following statements are equivalent:

(1) $p \dot{\wedge} q \in O P(V)$.

(2) $p \dot{\vee} q \in O P(V)$.

(3) $p^{\prime} \dot{\wedge} q^{\prime} \in O P(V)$.

(4) $p^{\prime} \dot{\vee} q^{\prime} \in O P(V)$

Proof. (1) implies (2): Let $p \dot{\wedge} q \in O P(V)$. By Proposition 5.9, $p \dot{\wedge} q \leq p$ so that by Corollary 5.7, $p-(p \dot{\wedge} q) \in O P(V)$. Now, $(p \dot{\wedge} q)+(p-p \dot{\wedge} q)=p \in O P(V)$ so that by Proposition 5.5, we get $p \dot{\wedge} q \perp(p-p \dot{\wedge} q)$. Since $(p-p \dot{\wedge} q) \perp(q-p \dot{\wedge} q)$ by Proposition 5.5, the additivity of $\perp$ yields, $q \perp(p-p \dot{\wedge} q)$. Again invoking Propositions 5.5 and 5.9, we may conclude that $p \dot{\vee} q=q+p-p \dot{\wedge} q \in O P(V)$.

(2) implies (3): We have $p^{\prime} \dot{\wedge} q^{\prime}=e-(p \dot{\vee} q) \in O P(V)$, if $p \dot{\vee} q \in O P(V)$.

Now, (3) implies (4) by step one and (4) implies (1) by step two.

Theorem 5.11. Let $V$ be an absolute order unit space and let $p, q \in O P(V)$. Then the following statements are equivalent:

(1) $p \dot{\wedge} q \in O P(V)$;

(2) $p$ is absolutely compatible with $q$;

(3) $p \dot{\wedge} q, p \dot{\wedge} q^{\prime} \in O P(V)$.

Proof. Note that (3) is stronger than (1).

(1) implies (2): Let $p \dot{\wedge} q \in O P(V)$. Then by Theorem 5.10, $p^{\prime} \dot{\wedge} q^{\prime} \in O P(V)$. Now

$$
\begin{aligned}
p \dot{\wedge} q+p^{\prime} \dot{\wedge} q^{\prime} & =\frac{1}{2}\left\{p+q-|p-q|+p^{\prime}+q^{\prime}-\left|p^{\prime}-q^{\prime}\right|\right\} \\
& =\frac{1}{2}\{2 e-2|p-q|\} \\
& =e-|p-q|
\end{aligned}
$$

for $p+p^{\prime}=e, q+q^{\prime}=e$ and $p^{\prime}-q^{\prime}=e-p-e+q=q-p$. Similarly,

$$
\begin{aligned}
p \dot{\wedge} q-p^{\prime} \dot{\wedge} q^{\prime} & =\frac{1}{2}\left\{p+q-|p-q|-p^{\prime}-q^{\prime}+\left|p^{\prime}-q^{\prime}\right|\right\} \\
& =\frac{1}{2}\{2 p+2 q-2 e\} \\
& =p+q-e .
\end{aligned}
$$

Since $p \dot{\wedge} q \leq p$ and $p^{\prime} \dot{\wedge} q^{\prime} \leq p^{\prime}$, and since $p \perp p^{\prime}$, we have $p \dot{\wedge} q \perp p^{\prime} \dot{\wedge} q^{\prime}$. Thus $\left|p \dot{\wedge} q-p^{\prime} \dot{\wedge} q^{\prime}\right|=p \dot{\wedge} q+p^{\prime} \dot{\wedge} q^{\prime}$ so that $|p+q-e|=e-|p-q|$. 
(2) implies (3): Let $|p-q|+\left|p-q^{\prime}\right|=e$. Then

$$
\begin{aligned}
p \dot{\wedge} q & =\frac{1}{2}\{p+q-|p-q|\} \\
& =\frac{1}{2}\left\{p+q-e+\left|p-q^{\prime}\right|\right\} \\
& =\frac{1}{2}\left\{p-q^{\prime}+\left|p-q^{\prime}\right|\right\} \in V^{+} .
\end{aligned}
$$

Similarly, $p \dot{\wedge} q^{\prime} \in V^{+}$. Further, as $p \dot{\wedge} q \leq q, p \dot{\wedge} q^{\prime} \leq q^{\prime}$ and $q \perp q^{\prime}$, we get that $p \dot{\wedge} q \perp p \dot{\wedge} q^{\prime}$. Thus by Proposition 5.6, $p \dot{\wedge} q, p \dot{\wedge} q^{\prime} \in O P(V)$ for

$$
\begin{aligned}
p \dot{\wedge} q+p \dot{\wedge} q^{\prime} & =\frac{1}{2}\left\{p+q-|p-q|+p+q^{\prime}-\left|p-q^{\prime}\right|\right\} \\
& =\frac{1}{2}\{2 p+e-e\}=p \in O P(V) .
\end{aligned}
$$

Remark 5.12. Let $p, q \in O P(V)$ such that $p \dot{\wedge} q \in O P(V)$. Then $r \dot{\wedge} s, r \dot{\vee} s \in$ $O P(V)$ whenever $r, s \in\left\{p, q, p^{\prime}, q^{\prime}\right\}$.

Proposition 5.13. Let $V$ be an absolute order unit space and let $p, q \in O P(V)$. Then $p \dot{\wedge} q \in O P(V)$ if and only if $|p-q| \in O P(V)$ with $|p-q| \leq p+q$.

Proof. First, let $p \dot{\wedge} q \in O P(V)$. Then $p \dot{\vee} q \in O P(V)$ by Theorem 5.10. Also, $p \dot{\wedge} q \leq p \dot{\vee} q$ with $|p-q|=p \dot{\vee} q-p \dot{\wedge} q$ by Proposition 5.9. Thus $|p-q| \in O P(V)$ by Corollary 5.7. Further, as $p \dot{\wedge} q \geq 0$, we have $|p-q| \leq p+q$.

Conversely, let $|p-q| \in O P(V)$ with $|p-q| \leq p+q$. Then $p \dot{\wedge} q \geq 0$ so that $p \dot{\wedge} q \in[0, e]$ for $p \dot{\wedge} q \leq p \leq e$. Next, $(p-p \dot{\wedge} q)+(q-p \dot{\wedge} q)=|p-q| \in O P(V)$. Also, by Proposition 5.9, we have $(p-p \dot{\wedge} q) \perp(q-p \dot{\wedge} q)$. Thus by Proposition 5.6, we get that $(p-p \dot{\wedge} q) \in O P(V)$. Since $p \dot{\wedge} q \geq 0$, we have $(p-p \dot{\wedge} q) \leq p$. Thus by Corollary 5.7, we have $p \dot{\wedge} q=p-(p-p \dot{\wedge} q) \in O P(V)$.

Theorem 5.14. Let $V$ be an absolute order unit space and let $p, q \in O P(V)$ with $p \dot{\wedge} q \in O P(V)$. Then $\inf _{O P(V)}\{u, v\}$ exists and is equal to $p \dot{\wedge} q$.

Proof. Let $r \leq p$ and $r \leq q$ for some $r \in O P(V)$. Then $r \perp p^{\prime}$ and $r \perp q^{\prime}$. Thus by the additivity of $\perp$, we get $r \perp\left(p^{\prime}+q^{\prime}\right)$. Since $p \dot{\wedge} q \in O P(V)$, we have $p^{\prime} \dot{\wedge} q^{\prime}, p^{\prime} \dot{\vee} q^{\prime} \in O P(V)$ by Theorem 5.10. Thus using Proposition 5.9, we see that $0 \leq p^{\prime} \dot{\vee} q^{\prime}=p^{\prime}+q^{\prime}-\left(p^{\prime} \dot{\wedge} q^{\prime}\right) \leq p^{\prime}+q^{\prime}$ so that $r \perp\left(p^{\prime} \dot{\vee} q^{\prime}\right)$. As $r, p^{\prime} \dot{\vee} q^{\prime} \in O P(V)$, by Proposition 5.5, we have $r+p^{\prime} \dot{\vee} q^{\prime} \leq e$. But $p^{\prime} \dot{\vee} q^{\prime}=e-(p \dot{\wedge} q)$ so that $r \leq p \dot{\wedge} q$. Now as $p \dot{\wedge} q \leq p, q$, we conclude that $\inf _{O P(V)}\{u, v\}$ exists and is equal to $p \dot{\wedge} q$.

Corollary 5.15. Let $V$ be an absolute order unit space and let $p, q \in O P(V)$ with $p \dot{\vee} q \in O P(V)$. Then $\sup _{O P(V)}\{p, q\}$ exists and is equal to $p \dot{\vee} q$.

Proposition 5.16. Let $V$ be an absolute order unit space and let $p, q \in O P(V)$ with $p \dot{\wedge} q \in O P(V)$ so that $r=|p-q| \in O P(V)$. Then the set $S:=\left\{0, e, p, q, r, p^{\prime}, q^{\prime}, r^{\prime}\right\}$ is closed under the binary operation $(u, v) \mapsto|u-v|$. 
Proof. Since $p \dot{\wedge} q \in O P(V)$, we have that $|e-p-q|=e-|p-q|=e-r$ and that $p \dot{\wedge} q^{\prime}, p^{\prime} \dot{\wedge} q, p^{\prime} \dot{\wedge} q^{\prime} \in O P(V)$. Also $p \dot{\wedge} q+p \dot{\wedge} q^{\prime}=p$. Since $p \dot{\wedge} q \leq q$ and since $p \dot{\wedge} q^{\prime} \leq q^{\prime}$, we have $p \dot{\wedge} q \perp p \dot{\wedge} q^{\prime}$ so that $\left|p \dot{\wedge} q-p \dot{\wedge} q^{\prime}\right|=p \dot{\wedge} q+p \dot{\wedge} q^{\prime}=p$. also

$$
2\left(p \dot{\wedge} q-p \dot{\wedge} q^{\prime}\right)=(p+q-r)-\left(p+q^{\prime}-r^{\prime}\right)=2(q-r)
$$

so that $p=|q-r|$. Now, by symmetry we can get $q=|p-r|$. In a similar way, we can calculate $|u-v|$ for any $u, v \in S$ to complete the proof.

\section{EXPANDING THE SCOPE}

In this section, we shall examine absolute compatibility of an order projection with a general positive element. First we note that order projections in an absolute order unit space have the following 'norming' property.

Proposition 6.1. Let $V$ be an absolute order unit space.

(1) If $p \in O P(V)$, then $p$ has the absolute order unit property in $V$. Dually, $e-p$ also has the absolute order unit property in $V$.

(2) Let $u \in[0, e]$ and assume that $u$ and $e-u$ have the absolute order unit property in $V$. Then $u \perp_{\infty}^{a}(e-u)$.

Proof. (1): First, assume that $p \in O P(V)$. Let $k p \pm v \in V^{+}$for some $k>0$. Set $v_{1}=\frac{1}{2}(k p+v)$ and $v_{2}=\frac{1}{2}(k p-v)$. Then $v_{1}, v_{2} \in V^{+}$with $v_{1}-v_{2}=v$ and $v_{1}+v_{2}=k p$. As $p \in O P(V)$, we have $p \perp(e-p)$ and consequently, $v_{1} \perp(e-p)$ and $v_{2} \perp(e-p)$. Thus $|v|=\left|v_{1}-v_{2}\right| \perp(e-p)$. Since $V$ is an absolute order unit space, we further have $|v| \perp_{\infty}^{a}(e-p)$ so that $\left\|\left|\||v|\|^{-1}\right| v \mid+(e-p)\right\|=1$. Now, for $f \in S(V)$, we have

$$
1=\|\| v\left\|^{-1}|v|+(e-p)\right\| \geq f\left(\|v\|^{-1}|v|+(e-p)\right)=1-f\left(p-\|v\|^{-1}|v|\right) .
$$

Thus $f\left(p-\|v\|^{-1}|v|\right) \geq 0$ for all $f \in S(V)$ so that $|v| \leq\|v\| p$. In other words, $p$ has the absolute order unit property in $V$.

(2): Conversely, let $u \in[0, e]$ and assume that $u$ and $e-u$ have the (absolute) order unit property in $V$. Let $0 \leq v \leq u$ and $0 \leq w \leq e-u$. By the order unit property of $u$ and $e-u$ we have $v \leq\|v\| u$ and $w \leq\|w\|(e-u)$. Thus

$$
0 \leq\|v\|^{-1} v+\|w\|^{-1} w \leq u+(e-u)=e
$$

so that \|\|$v\left\|^{-1} v+\right\| w\left\|^{-1} w\right\| \leq 1$. Now

$$
1=\|\| v\left\|^{-1} v\right\| \leq\|\| v\left\|^{-1} v+\right\| w\left\|^{-1} w\right\| \leq 1
$$

so that $u \perp_{\infty}^{a}(e-u)$.

Remark 6.2. (1) Let an absolute order unit space $(V, e)$ satisfy $\left(O . \perp_{\infty} .2\right)$. Then $p \in O P(V)$ if and only if $p \in[0, e]$ and both $p$ and $e-p$ satisfy the absolute order unit property.

(2) Let $(V, e)$ be an order unit space and let $u \in V^{+}$has the order unit property in $V$. Then $\left(V_{u}, u\right)$ is an order unit space. In this case, $u$ and $e$ determine the same norm in $V_{u}$. Thus $\left(V_{u}, u\right)$ is an 'order unit ideal' of $V$. 
(3) Let $(V, e)$ be an absolute order unit space and assume that $u \in V^{+}$has the absolute order unit property in $V$. Then $\left(V_{u}, u\right)$ is an absolute order unit space. In particular, if $p \in O P(V)$, then $\left(V_{p}, p\right)$ is an absolute order unit space and in this case,

$$
O P\left(V_{p}\right)=\{q \in O P(V): q \leq p\}=O P(V) \cap V_{p} .
$$

Proposition 6.3. Let $(V, e)$ be an order unit space and let $u \in[0, e]$. If $u$ has the order unit property in $V$, then it is an extreme point in $[0, e]$.

Proof. Let $u=\alpha v+(1-\alpha) w$ for some $v, w \in[0, e]$ and $0<\alpha<1$. Then $0 \leq \alpha v \leq u$. Since $u$ has the order unit property in $V$, we get, $v \leq\|v\| u \leq u$. Similarly, $0 \leq w \leq u$. Set $u-v=v_{1}$ and $u-w=w_{1}$. Then

$$
0=u-(\alpha v+(1-\alpha) w)=\alpha v_{1}+(1-\alpha) w_{1} .
$$

Since $V^{+}$is proper and $0<\alpha<1$, we get $v_{1}=0=w_{1}$ so that $v=u=w$.

The absolute compatibility between an order projection $p \in O P(V)$ and an arbitrary element $u \in[0, e]$ is related to $V_{p}+V_{p^{\prime}}$ which we describe below. Let $\left(V_{1}, e_{1}\right)$ and $\left(V_{2}, e_{2}\right)$ be any two absolute order unit spaces. Consider

$$
V=V_{1} \times V_{2} ; \quad V^{+}=V_{1}^{+} \times V_{2}^{+} ; \text {and } e=\left(e_{1}, e_{2}\right) .
$$

Then $(V, e)$ becomes an absolute order unit space in a canonical way. Further, $V$ is unitally and isometrically order isomorphic to $V_{1} \oplus_{\infty} V_{2}$.

Proposition 6.4. Let $(V, e)$ be an absolute order unit normed space and let $p, q \in O P(V)$ with $p+q \leq e$. Then $V_{p}+V_{q}$ is an absolute order smooth $\infty$-normed subspace of $(V, e)$ and is isometrically order isomorphic to $V_{p} \oplus_{\infty} V_{q}$. In particular,for $x \in V_{p}$ and $y \in V_{q}$, we have $(x+y)^{+}=x^{+}+y^{+}$and $(x+y)^{-}=x^{-}+y^{-}$ so that $|x+y|=|x|+|y|$.

Proof. Let $u \in V_{p}$ and $v \in V_{q}$. Then $u^{+}, u^{-} \in V_{p}^{+}$and $v^{+}, v^{-} \in V_{q}^{+}$. As $p+q \leq e$, we have $p \perp_{\infty}^{a} q$ and consequently, we may conclude that $u^{+}, u^{-}, v^{+}$ and $v^{-}$are absolutely $\infty$-orthogonal to each other. Now, by the additivity, we have $\left(u^{+}+v^{+}\right) \perp_{\infty}^{a}\left(u^{-}+v^{-}\right)$. Thus as $u+v=\left(u^{+}+v^{+}\right)-\left(u^{-}+v^{-}\right)$, we get that $(u+v)^{+}=u^{+}+v^{+} ;(u+v)^{-}=u^{-}+v^{-}$so that $|u+v|=|u|+|v|$. Therefore, $V_{p}+V_{q}$ is an absolute ordered space. It also follows that $V_{p} \cap V_{q}=\{0\}$. Finally, for $u \in V_{p}$ and $v \in V_{q}$ we note that

$$
\begin{aligned}
\|u+v\| & =\max \left\{\left\|(u+v)^{+}\right\|,\left\|(u+v)^{-}\right\|\right\} \\
& =\max \left\{\left\|u^{+}+v^{+}\right\|,\left\|u^{-}+v^{-}\right\|\right\} \\
& =\max \left\{\left\|u^{+}\right\|,\left\|v^{+}\right\|,\left\|u^{-}\right\|,\left\|v^{-}\right\|\right\} \\
& =\max \{\|u\|,\|v\|\}
\end{aligned}
$$

which completes the proof.

Theorem 6.5. Let $(V, e)$ be an absolute order unit normed space and let $p, q \in$ $O P(V)$ with $p+q \leq e$. Then for $w \in V$, the following statements are equivalent:

(1) $w \in V_{p}^{+}+V_{q}^{+}$with $\|w\| \leq 1$;

(2) $w=p \dot{\wedge} w+q \dot{\wedge} w$; 
(3) $|p-w|+|q-w|=p+q$.

Proof. (1) implies (2): Let $w \in V_{p}^{+}+V_{q}^{+}$with $\|w\| \leq 1$. By Proposition 6.4, there exists a unique pair $w_{p} \in V_{p}^{+}$and $w_{q} \in V_{q}^{+}$such that $w=w_{p}+w_{q}$. As $\|w\| \leq 1$, we get that $w_{p} \leq p$ and $w_{q} \leq q$ so that $0 \leq p-w_{p} \leq p$. Since $p+q \leq e$, we have $p \perp_{\infty}^{a} q$ and consequently, $\left(p-w_{p}\right) \perp_{\infty}^{a} w_{q}$. Thus

$$
|p-w|=\left|p-w_{p}-w_{q}\right|=p-w_{p}+w_{q}
$$

Therefore,

$$
u \dot{\wedge} w=\frac{1}{2}(p+w-|p-w|)=w_{p}
$$

Similarly, $q \dot{\wedge} w=w_{q}$ so that $w=p \dot{\wedge} w+q \dot{\wedge} w$.

(2) implies (3): Let $w=p \dot{\wedge} w+q \dot{\wedge} w$. Then

$$
2 w=p+w-|p-w|+q+w-|q-w|
$$

so that $|p-w|+|q-w|=p+q$.

(3) implies (1): Finally, let $|p-w|+|q-w|=p+q$. Consider the $\perp_{\infty^{-}}^{a}$ decompositions $p-w=u_{1}-u_{2}$ and $q-w=v_{1}-v_{2}$ in $V^{+}$. Then $|p-w|=u_{1}+u_{2}$ and $|q-w|=v_{1}+v_{2}$. Now it follows that

$$
u_{1}+u_{2}+v_{1}+v_{2}=p+q
$$

and

$$
u_{1}-u_{2}+v_{1}-v_{2}=p+q-2 w .
$$

Therefore, $w=u_{2}+v_{2}$ and consequently, $p=u_{1}+v_{2}$ and $q=v_{1}+u_{2}$. Now, as $0 \leq v_{2} \leq p$ and $0 \leq u_{2} \leq q$, we have $w \in V_{p}^{+}+V_{q}^{+}$. Further as $p \perp_{\infty}^{a} q$, we have $u_{2} \perp_{\infty}^{a} v_{2}$. Thus

$$
\|w\|=\left\|u_{2}+v_{2}\right\|=\max \left\{\left\|u_{2}\right\|,\left\|v_{2}\right\|\right\} \leq \max \{\|p\|,\|q\|\} \leq 1 .
$$

Remark 6.6. It follows from the proof of Theorem 6.5 that if $p, q \in O P(V)$ with $p+q \leq e$ and if $w \in V_{p}^{+}+V_{q}^{+}$with $\|w\| \leq 1$, then the $p$ and $q$ "components" of $w$ are $p \dot{\wedge} w$ and $q \dot{\wedge} w$ respectively. More generally, if $w \in V_{p}^{+}+V_{q}^{+}$, then the $p$ and $q$ "components" of $w$ are $(k p) \dot{\wedge} w=(\|w\| p) \dot{\wedge} w$ and $(k q) \dot{\wedge} w=(\|w\| q) \dot{\wedge} w$ respectively for any $k \geq\|w\|$.

We shall write $A C(p):=V_{p}+V_{p^{\prime}}$ so that $A C(p)^{+}:=\left(V_{p}+V_{p^{\prime}}\right) \cap V^{+}=V_{p}^{+}+V_{p^{\prime}}^{+}$.

Corollary 6.7. Let $(V, e)$ be an absolute order unit normed space, $p \in O P(V)$ and $u \in[0, e]$. Then $u$ is absolutely compatible with $p$ if and only if $u \in A C(p)^{+}$.

Proposition 6.8. Let $(V, e)$ be an absolute order unit normed space, $p \in O P(V)$ and $u \in[0, e]$ and assume that $u$ is absolutely compatible with $p$. Then

(1) $\inf \{u, p\}$ exists in $[0, e]$ and is equal to $u \dot{\wedge} p$; and

(2) $\sup \{u, p\}$ exists in $[0, e]$ and is equal to $u \dot{\vee} p$. 
Proof. (1): By the definition, we have $u \dot{\wedge} p \leq u$ and $u \dot{\wedge} p \leq p$. As $u$ is absolutely compatible with $p$, we have $u \dot{\wedge} p, u \dot{\wedge} p^{\prime} \in V^{+}$with $u=u \dot{\wedge} p+u \dot{\wedge} p^{\prime}$. Next, let $w \in V^{+}$such that $w \leq u$ and $w \leq p$. Then $w \in V_{p}^{+}$. Also, $u \dot{\wedge} p \in V_{p}^{+}$so that $u \dot{\wedge} p-w \in V_{p}$. Further $u \dot{\wedge} p^{\prime} \in V_{p^{\prime}}^{+}$. Thus by Theorem 6.5, we get

$$
\begin{aligned}
u-w=|u-w| & =\left|(u \dot{\wedge} p-w)+\left(u \dot{\wedge} p^{\prime}\right)\right| \\
& =|u \dot{\wedge} p-w|+\left|u \dot{\wedge} p^{\prime}\right| \\
& =|u \wedge p-w|+u \wedge p^{\prime}
\end{aligned}
$$

so that $u \dot{\wedge} p-w=|u \dot{\wedge} p-w| \in V^{+}$. Hence $u \dot{\wedge} p=\inf \{u, p\}$.

(2): As $u$ is absolutely compatible with $p$, we have $e-u$ is absolutely compatible with $e-p$. Thus as in (1), we may conclude that $(e-u) \dot{\wedge}(e-p)=\inf \{e-u, e-p\}$. Now it follows that

$$
u \dot{\vee} p=e-((e-u) \dot{\wedge}(e-v))=e-\inf \{e-u, e-v\}=\sup \{u, p\} .
$$

Theorem 6.9. Let $(V, e)$ be an absolute order unit normed space and let $p \in$ $O P(V)$. Then for $u_{1}, \ldots, u_{n} \in A C(p)^{+}$, we have

$$
\left(\sum_{i=1}^{n} u_{i}\right) \dot{\wedge}\left(\sum_{i=1}^{n}\left\|u_{i}\right\| p\right)=\sum_{i=1}^{n}\left(u_{i} \dot{\wedge}\left\|u_{i}\right\| p\right)
$$

and

$$
\left(\sum_{i=1}^{n} u_{i}\right) \dot{\vee}\left(\sum_{i=1}^{n}\left\|u_{i}\right\| p\right)=\sum_{i=1}^{n}\left(u_{i} \dot{\vee}\left\|u_{i}\right\| p\right) .
$$

Proof. First, let $u_{1}, u_{2} \in A C(p)^{+}$with $\left\|u_{i}\right\| \leq 1, i=1,2$ and assume that $\alpha \in$ $[0,1]$. Then by Corollary 6.7 , we have $u_{i}=u_{i} \dot{\wedge} p+u_{i} \dot{\wedge} p^{\prime}$ with $u_{i} \dot{\wedge} p, u_{i} \dot{\wedge} p^{\prime} \in V^{+}$ for $i=1,2$. Thus

$$
\begin{aligned}
& \left|p-\alpha u_{1}-(1-\alpha) u_{2}\right| \\
& \quad=\left|\left\{\alpha\left(p-u_{1} \dot{\wedge} p\right)+(1-\alpha)\left(p-u_{2} \dot{\wedge} p\right)\right\}-\left\{\alpha\left(u_{1} \dot{\wedge} p^{\prime}\right)+(1-\alpha)\left(u_{2} \dot{\wedge} p^{\prime}\right)\right\}\right| \\
& \quad=\left\{\alpha\left(p-u_{1} \dot{\wedge} p\right)+(1-\alpha)\left(p-u_{2} \dot{\wedge} p\right)\right\}+\left\{\alpha\left(u_{1} \dot{\wedge} p^{\prime}\right)+(1-\alpha)\left(u_{2} \dot{\wedge} p^{\prime}\right)\right\}
\end{aligned}
$$

for

$$
\begin{gathered}
0 \leq \alpha\left(p-u_{1} \dot{\wedge} p\right)+(1-\alpha)\left(p-u_{2} \dot{\wedge} p\right) \leq p \\
0 \leq \alpha\left(u_{1} \dot{\wedge} p^{\prime}\right)+(1-\alpha)\left(u_{2} \dot{\wedge} p^{\prime}\right) \leq p^{\prime}
\end{gathered}
$$

and $p \perp_{\infty}^{a} p^{\prime}$. On simplifying, we get

$$
\left|\alpha u_{1}+(1-\alpha) u_{2}-p\right|=\alpha u_{1}+(1-\alpha) u_{2}+p-2\left\{\alpha\left(u_{1} \dot{\wedge} p\right)+(1-\alpha)\left(u_{2} \dot{\wedge} p\right)\right\} .
$$

Now it follows that

$$
\left(\alpha u_{1}+(1-\alpha) u_{2}\right) \dot{\wedge} p=\alpha\left(u_{1} \dot{\wedge} p\right)+(1-\alpha)\left(u_{2} \dot{\wedge} p\right)
$$

and that

$$
\left(\alpha u_{1}+(1-\alpha) u_{2}\right) \dot{\vee} p=\alpha\left(u_{1} \dot{\vee} p\right)+(1-\alpha)\left(u_{2} \dot{\vee} p\right)
$$


By a standard technique, now we can now show that this fact also holds for $n$ elements: For $u_{1}, \ldots, u_{n} \in A C(p)^{+}$with $\left\|u_{i}\right\| \leq 1, i=1, \ldots, n$ and positive real numbers $\alpha_{1}, \ldots, \alpha_{n}$ with $\sum_{i=1}^{n} \alpha_{i}=1$, we have

$$
\text { (!) }\left(\sum_{i=1}^{n} \alpha_{i} u_{i}\right) \dot{\wedge} p=\sum_{i=1}^{n} \alpha_{i}\left(u_{i} \dot{\wedge} p\right)
$$

and

$$
\text { (!!) }\left(\sum_{i=1}^{n} \alpha_{i} u_{i}\right) \dot{\vee} p=\sum_{i=1}^{n} \alpha_{i}\left(u_{i} \dot{\vee} p\right) \text {. }
$$

Finally, let $u_{1}, \ldots, u_{n} \in A C(p)^{+} \backslash\{0\}$. Set $w_{i}=\left\|u_{i}\right\|^{-1} u_{i}$ so that $\left\|w_{i}\right\|=1, i=$ $1, \ldots, n$. Then for $k=\sum_{i=1}^{n}\left\|u_{i}\right\|$, using (!), we get

$$
\begin{aligned}
\left(\sum_{i=1}^{n} u_{i}\right) \dot{\wedge}(k p) & =k\left(\left(\sum_{i=1}^{n} \frac{\left\|u_{i}\right\|}{k} w_{i}\right) \dot{\wedge} p\right) \\
& =k\left(\sum_{i=1}^{n} \frac{\left\|u_{i}\right\|}{k}\left(w_{i} \dot{\wedge} p\right)\right) \\
& =\sum_{i=1}^{n}\left\|u_{i}\right\|\left(w_{i} \dot{\wedge} p\right) \\
& =\sum_{i=1}^{n}\left(u_{i} \dot{\wedge}\left\|u_{i}\right\| p\right)
\end{aligned}
$$

which proves (1). Similarly, using (!!), we may get (2).

Let $(V, e)$ be an absolute order unit normed space and let $\left\{p_{i}: i \in I\right\} \subset O P(V)$. We shall write $A C\left(p_{i} ; i \in I\right)$ for $\cap_{i \in I} A C\left(p_{i}\right)$.

Theorem 6.10. Let $(V, e)$ be an absolute order unit normed space and let $v \in$ $A C^{+}(p, q)$ for some $p, q \in O P(V)$ such that $p+q \leq e$. Set $r=e-p-q$ so that $r \in O P(V)$. Then $v \in A C^{+}(r)$ and $v=v \dot{\wedge} p+v \dot{\wedge} q+v \dot{\wedge} r$.

Proof. We may assume that $\|v\| \leq 1$. As $v \in A C^{+}(p)$, we have

(i) $\quad v=v \dot{\wedge} p+v \dot{\wedge} p^{\prime}$.

Now, $p \leq e-q=q^{\prime}$ so that $v \dot{\wedge} p \in V_{p}^{+} \subset V_{q^{\prime}} \subset A C^{+}(q)$. Also, $v \in A C^{+}(q)$ so that $v \dot{\wedge} p^{\prime}=v-v \dot{\wedge} p \in A C(q)$. But $v \dot{\wedge} p^{\prime} \in V^{+}$so that $v \dot{\wedge} p^{\prime} \in A C^{+}(q)$. As $\left\|v \dot{\wedge} p^{\prime}\right\| \leq\|v\| \leq 1$ we get

(ii) $\quad v \dot{\wedge} p^{\prime}=\left(v \dot{\wedge} p^{\prime}\right) \dot{\wedge} q+\left(v \dot{\wedge} p^{\prime}\right) \dot{\wedge} q^{\prime}$.

We show that $\left(v \dot{\wedge} p^{\prime}\right) \dot{\wedge} q=v \dot{\wedge} q$. We have

$$
\begin{aligned}
|v-q| & =\left|v \dot{\wedge} p+\left(v \dot{\wedge} p^{\prime}\right) \dot{\wedge} q+\left(v \dot{\wedge} p^{\prime}\right) \dot{\wedge} q^{\prime}-q\right| \\
& =\left|\left\{q-\left(v \dot{\wedge} p^{\prime}\right) \dot{\wedge} q\right\}-\left\{v \dot{\wedge} p+\left(v \dot{\wedge} p^{\prime}\right) \dot{\wedge} q^{\prime}\right\}\right|
\end{aligned}
$$

Now, as $0 \leq\left(v \dot{\wedge} p^{\prime}\right) \dot{\wedge} q \leq q$, we have $0 \leq q-\left(v \dot{\wedge} p^{\prime}\right) \dot{\wedge} q \leq q$. Thus, as $0 \leq$ $v \dot{\wedge} p \leq p, 0 \leq\left(v \dot{\wedge} p^{\prime}\right) \dot{\wedge} q^{\prime} \leq q^{\prime}, q \perp p$ and $q \perp q^{\prime}$; we get $\left\{q-\left(v \dot{\wedge} p^{\prime}\right) \dot{\wedge} q^{\prime}\right\} \perp$ 
$v \dot{\wedge} p$ and $\left\{q-\left(v \dot{\wedge} p^{\prime}\right) \dot{\wedge} q^{\prime}\right\} \perp\left(v \dot{\wedge} p^{\prime}\right) \dot{\wedge} q^{\prime}$. Now, by the additivity of $\perp$, we get $\left\{q-\left(v \dot{\wedge} p^{\prime}\right) \dot{\wedge} q^{\prime}\right\} \perp\left\{v \dot{\wedge} p+\left(v \dot{\wedge} p^{\prime}\right) \dot{\wedge} q^{\prime}\right\}$. Thus

$$
|v-q|=\left\{q-\left(v \dot{\wedge} p^{\prime}\right) \dot{\wedge} q^{\prime}\right\}+v \dot{\wedge} p+\left(v \dot{\wedge} p^{\prime}\right) \dot{\wedge} q^{\prime}
$$

so that

$$
\begin{aligned}
2(v \dot{\wedge} q) & =v+q-|v-q| \\
& =v+q-\left\{q-\left(v \dot{\wedge} p^{\prime}\right) \dot{\wedge} q^{\prime}\right\}-v \dot{\wedge} p-\left(v \dot{\wedge} p^{\prime}\right) \dot{\wedge} q^{\prime} \\
& =2\left\{\left(v \dot{\wedge} p^{\prime}\right) \dot{\wedge} q\right\} .
\end{aligned}
$$

Thus by (i) and (ii), we have

(iii) $\quad v=v \dot{\wedge} p+v \dot{\wedge} q+\left(v \dot{\wedge} p^{\prime}\right) \dot{\wedge} q^{\prime}$.

Now, interchanging $p$ and $q$, we may conclude that

(iv) $\quad\left(v \dot{\wedge} p^{\prime}\right) \dot{\wedge} q^{\prime}=\left(v \dot{\wedge} q^{\prime}\right) \dot{\wedge} p^{\prime}$

Finally, we shall show that $\left(v \dot{\wedge} p^{\prime}\right) \dot{\wedge} q^{\prime}=v \dot{\wedge} r$. Let us quickly note that $v \dot{\wedge} p \leq p$ and $v \dot{\wedge} q \leq q$ so that $v \dot{\wedge} p+v \dot{\wedge} q \in V_{p+q}^{+}$. Also, $\left(v \dot{\wedge} p^{\prime}\right) \dot{\wedge} q^{\prime} \leq p^{\prime}$ and $\left(v \dot{\wedge} p^{\prime}\right) \dot{\wedge} q^{\prime} \leq q^{\prime}$ so that $\left(v \dot{\wedge} p^{\prime}\right) \dot{\wedge} q^{\prime} \leq p^{\prime} \dot{\wedge} q^{\prime}=(p+q)^{\prime}=r$. Thus $\left(v \dot{\wedge} p^{\prime}\right) \dot{\wedge} q^{\prime} \in V_{r}^{+}$. Now, it follows from (iii) that $v \in A C^{+}(r)$. Therefore,

$$
|v-r|+|v+r-e|=e \text {. }
$$

Now,

$$
\begin{aligned}
|v-p-q| & =\left|(p-v \dot{\wedge} p)+(q-v \dot{\wedge} q)-\left(v \dot{\wedge} p^{\prime}\right) \dot{\wedge} q^{\prime}\right| \\
& =(p-v \dot{\wedge} p)+(q-v \dot{\wedge} q)+\left(v \dot{\wedge} p^{\prime}\right) \dot{\wedge} q^{\prime}
\end{aligned}
$$

for $0 \leq(p-v \dot{\wedge} p)+(q-v \dot{\wedge} q) \leq p+q$ and $0 \leq\left(v \dot{\wedge} p^{\prime}\right) \dot{\wedge} q^{\prime} \leq(p+q)^{\prime}$. Thus

$$
\begin{aligned}
2(v \dot{\wedge} r) & =v+r-|v-r| \\
& =v+e-(p+q)-e+|v-(p+q)| \quad(\text { by }(\mathrm{v})) \\
& =v-(p+q)+(p-v \dot{\wedge} p)+(q-v \dot{\wedge} q)+\left(v \dot{\wedge} p^{\prime}\right) \dot{\wedge} q^{\prime} \\
& =2\left\{\left(v \dot{\wedge} p^{\prime}\right) \dot{\wedge} q^{\prime}\right\} .
\end{aligned}
$$

Putting it in (iii), we get the result.

Corollary 6.11. Let $(V, e)$ be an absolute order unit normed space and let $w \in$ $A C^{+}(p, q)$ for some $p, q \in O P(V)$.

(1) If $p+q \leq e$, then $w \in A C^{+}(q+p)$.

(2) If $p \leq q$, then $w \in A C^{+}(q-p)$.

Remark 6.12. Let $(V, e)$ be an absolute order unit normed space and let $w \in$ $A C^{+}\left(p_{i} ; 1 \leq i \leq n\right)$ for some $p_{1}, \ldots, p_{n} \in O P(V)$. The results of Corollary 6.11 may be generalized in the following way.

(1) If $p=p_{1}+\cdots+p_{n} \leq e$ so that $p \in A P(V)$ and that $p_{1}, \ldots, p_{n}, p^{\prime}$ are mutually $\perp_{\infty}^{a}$-orthogonal, we have $w \in V_{p_{1}}^{+}+V_{p_{2}}^{+}+\cdots+V_{p_{n}}^{+}+V_{p^{\prime}}^{+}$and $w \in A C^{+}\left(\sum_{i \in I} p_{i}\right)$ whenever $I \subset\{1, \ldots, n\}$.

(2) If $p_{1} \leq \ldots, \leq p_{n}$, then $w \in V_{p_{1}}^{+}+V_{p_{2}-p_{1}}^{+}+\cdots+V_{p_{n}-p_{n-1}}^{+}+V_{p_{n}^{\prime}}^{+}$and $w \in A C^{+}\left(p_{i+k}-p_{i}\right)$ whenever $1 \leq i, k \leq n$ with $i+k \leq n$. Also, in this case, $p_{1}, p_{2}-p_{1}, \ldots, p_{n}-p_{n-1}, p_{n}^{\prime} \in O P(V)$ are mutually $\perp_{\infty}^{a}$-orthogonal. 


\section{Spectral FAmily of order PROJECtions}

In this section we shall discuss spectral family of order projections for an element in an absolute order unit space $(V, e)$. For this purpose, we need the following concept.

\subsection{A hypothesis for $O P(V)$.}

In general, a $\mathrm{C}^{*}$-algebra may not have sufficiently many projections. However, in a von Neumann algebra $M, O P(M)$ always covers $M_{s a}$ in the following sense.

Definition 7.1. Let $(V, e)$ be an absolute order unit space. We say that $p \in$ $O P(V)$ covers an element $v \in V$, if $v \in V_{p}$ and $V_{p} \subset V_{q}$ whenever $v \in V_{q}$. In other words, $p$ exists as the least element (with respect to $O P(V)$ ) in the set

$$
O P_{v}(V):=\left\{r \in O P(V): v \in V_{r}\right\}=\left\{r \in O P(V): v \perp_{\infty}^{a}(e-r)\right\} .
$$

We say that $O P(V)$ covers $V$, if every element $v \in V$ has a cover in $O P(V)$ and $p_{1} \perp_{\infty}^{a} p_{2}$ whenever $p_{i}$ is the cover of $v_{i} \in V^{+}$in $O P(V)$ for $i=1,2$ with $v_{1} \perp_{\infty}^{a} v_{2}$.

The covering property also determines a lattice structure in $O P(V)$.

Proposition 7.2. Let $(V, e)$ be an absolute order unit space in which $O P(V)$ covers $V$. Then $O P(V)$ is a lattice in the order structure of $V$ restricted to $O P(V)$.

Proof. Let $p_{1}, p_{2} \in O P(V)$ and let $p \in O P(V)$ be the cover of $p_{1}+p_{2}$. Then $p_{1}+p_{2} \leq\left\|p_{1}+p_{2}\right\| p$ so that $p_{1} \leq p_{1}+p_{2} \leq\left\|p_{1}+p_{2}\right\| p$. Now, by the order unit property of $p$, we get $p_{1} \leq\left\|p_{1}\right\| p \leq p$. Similarly we can show that $p_{2} \leq p$. Next, let $p_{i} \leq q, i=1,2$ for some $q \in O P(V)$. Then $p_{i} \perp_{\infty}^{a} q^{\prime}, i=1,2$ so that $p_{1}+p_{2} \perp_{\infty}^{a} q^{\prime}$ by the additivity of $\perp_{\infty}^{a}$. Now by Lemma $4.5, p_{1}+p_{2} \leq\left\|p_{1}+p_{2}\right\| q$. Since $p$ covers $p_{1}+p_{2}$, we get $p \leq q$. Thus $p=\sup \left\{p_{1}, p_{2}\right\}$. Next, as $p_{1}^{\prime}, p_{2}^{\prime} \in O P(V)$, we have $r=\sup \left\{p_{1}^{\prime}, p_{2}^{\prime}\right\} \in O P(V)$. Now, by a well known trick, we can show that $\inf \left\{p_{1}, p_{2}\right\}=r^{\prime}$. Hence $O P(V)$ is a lattice.

\subsection{Construction of a spectral family.}

Definition 7.3. Let $(V, e)$ be an absolute order unit normed space and let $p \in$ $O P(V)$. Then every $v \in A C(p)$ has a unique decomposition

$$
v=v_{p}+v_{p^{\prime}}
$$

where $v_{p} \in V_{p}$ and $v_{p^{\prime}} \in V_{p^{\prime}}$. This decomposition will be referred as the $p$ decomposition of $v$ and we shall write $v_{p}=C_{p}(v)$ and $v_{p^{\prime}}=C_{p}^{\prime}(v)$ for all $v \in$ $A C(p)$. In particular, for $v \in A C(p)^{+}$, we have $C_{p}(v)=w \dot{\wedge}(\|w\| p)$ for all $p \in$ $O P(V)$. By Proposition 6.4, $C_{p}: A C(p) \rightarrow V_{p}$ is a $|\cdot|$-preserving surjective linear projection of norm one. Further, $C_{p}+C_{p}^{\prime}$ is the identity operator on $A C(u)$.

Proposition 7.4. Let $(V, e)$ be an absolute order unit space. If $p, q \in O P(V)$ with $p \leq q$, then $C_{p}, C_{q}, C_{p}^{\prime}$ and $C_{q}^{\prime}$ commute mutually when restricted to $A C(p, q)$. In this case, $C_{p} C_{q}=C_{p}$ and consequently, $C_{p} C_{q}^{\prime}=0, C_{p}^{\prime} C_{q}=C_{q}-C_{p}$ and $C_{p}^{\prime} C_{q}^{\prime}=C_{q}^{\prime}$ on $A C(p, q)$. 
Proof. Let $u \in A C(p, q)$. Then

$$
u=C_{p}(u)+C_{p}^{\prime}(u)=C_{q}(u)+C_{q}^{\prime}(u) .
$$

As $p \leq q$, we have $V_{p} \subset V_{q} \subset O C(q)$. Thus $C_{p}(u) \in A C(q)$ and consequently, $C_{p}^{\prime}(u)=u-C_{q}(u) \in O C(q)$. Now as $A C\left(p^{\prime}\right)=A C(p), A C\left(q^{\prime}\right)=$ $A C(q)$ and $q^{\prime} \leq p^{\prime}$ (for $p \leq q$ ), we may conclude, by the dual arguments, that $C_{p}(u), C_{q}(u), C_{p}^{\prime}(u), C_{q}^{\prime}(u) \in A C(p, q)$ whenever $u \in A C(p, q)$.

Next, note that for $u \in A C(p, q)$ we have $C_{p}(u) \in V_{p} \subset V_{q}$ so that $C_{q}\left(C_{p}(u)\right)=$ $C_{p}(u)$. Dually, $C_{p}^{\prime}\left(C_{q}^{\prime}(u)\right)=C_{q}^{\prime}(u)$ so that $C_{q} C_{p}=C_{p}$ and $C_{p}^{\prime} C_{q}^{\prime}=C_{q}^{\prime}$ on $A C(p, q)$. Now, if we recall that $C_{p}^{\prime}=I-C_{p}$ and that $C_{q}^{\prime}=I-C_{q}$ on $A C(p, q)$ where $I$ is the identity operator on $A C(p, q)$, the remaining facts can be verified in a routine way.

Throughout this subsection, we shall assume that $(V, e)$ is an absolute order unit space in which $O P(V)$ covers $V$ unless stated otherwise. We fix the following notations. Let $v \in V$ and $\alpha \in \mathbb{R}$. We write $c_{p}^{ \pm}(v, \alpha)$ for the cover of $(v-\alpha p)^{ \pm}$ in $O P(V)$ respectively, for any $p \in O P(V)$. For $p=e$, we shall simply write $c^{ \pm}(v, \alpha)$. Thus $c^{+}(v, \alpha) \perp c^{-}(v, \alpha)$. When $\alpha=0$, we shall simply write $c^{ \pm}(v)$ for $c^{ \pm}(v, 0)$. Also as $(-v,-\alpha)^{+}=(v, \alpha)^{-}$, we have, $c^{+}(-v,-\alpha)=c^{-}(v, \alpha)$.

Let $c(v)$ be the cover of $v$ in $O P(V)$. Then

$$
(v-\alpha e)^{+}=(v-\alpha c(v))^{+}+\left(-\alpha c(v)^{\prime}\right)^{+} \in V_{c(v)}^{+}+V_{c(v)^{\prime}}^{+}
$$

and

$$
(v-\alpha e)^{-}=(v-\alpha c(v))^{-}+\left(-\alpha c(v)^{\prime}\right)^{-} \in V_{c(v)}^{+}+V_{c(v)^{\prime}}^{+}
$$

Thus

$$
\begin{aligned}
(v-\alpha e)^{+} & =(v-\alpha c(v))^{+}, \quad \text { if } \quad \alpha \geq 0 \\
& =(v-\alpha c(v))^{+}-\alpha c(v)^{\prime}, \quad \text { if } \quad \alpha<0
\end{aligned}
$$

and

$$
\begin{aligned}
(v-\alpha e)^{-} & =(v-\alpha c(v))^{-}+\alpha c(v)^{\prime}, \quad \text { if } \quad \alpha>0 \\
& =(v-\alpha c(v))^{-}, \quad \text { if } \quad \alpha \leq 0 .
\end{aligned}
$$

Now, it follows that

$$
\begin{aligned}
c^{+}(v, \alpha) & =c_{c(v)}^{+}(v, \alpha) \quad \text { if } \quad \alpha \geq 0 \\
& =c_{c(v)}^{+}(v, \alpha)+c(v)^{\prime} \quad \text { if } \quad \alpha<0
\end{aligned}
$$

and

$$
\begin{aligned}
c^{-}(v, \alpha) & =c_{c(v)}^{-}(v, \alpha)+c(v)^{\prime} \quad \text { if } \quad \alpha>0 \\
& =c_{c(v)}^{-}(v, \alpha) \text { if } \quad \alpha \leq 0 .
\end{aligned}
$$

In a similar manner, we can further conclude that

$$
\begin{aligned}
c^{+}(v, \alpha) & =c_{c^{+}(v)}^{+}\left(v^{+}, \alpha\right) \quad \text { if } \quad \alpha \geq 0 \\
& =c_{c^{-}(v)}^{-}\left(v^{-},-\alpha\right)+c^{-}(v)^{\prime} \quad \text { if } \quad \alpha<0
\end{aligned}
$$


and

$$
\begin{aligned}
c^{-}(v, \alpha) & =c_{c^{+}(v)}^{-}\left(v^{+}, \alpha\right)+c^{+}(v)^{\prime} \quad \text { if } \quad \alpha>0 \\
& =c_{c^{-}(v)}^{+}\left(v^{-},-\alpha\right) \text { if } \quad \alpha \leq 0 .
\end{aligned}
$$

Remark 7.5. (1) For $v \in V^{+}, c^{+}(v, \alpha)=e$ whenever $\alpha<0$. Dually, $c^{-}(v, \alpha)=$ $e$ whenever $v \in-V^{+}$and $\alpha>0$.

(2) For $v \in V^{+}, c^{-}(v, \alpha)=0$ whenever $\alpha \leq 0$. Dually, $c^{+}(v, \alpha)=0$ whenever $v \in-V^{+}$and $\alpha \geq 0$.

Now we prove some results which show that $\left\{c^{-}(v, \alpha): \alpha \in \mathbb{R}\right\}$ and $\left\{c^{+}(v, \alpha)^{\prime}\right.$ : $\alpha \in \mathbb{R}\}$ are "spectral" families of projections for $v$.

Proposition 7.6. For $v \in V$, the family $\left\{c^{-}(v, \alpha), c^{+}(v, \alpha): \alpha \in \mathbb{R}\right\}$ has the following properties:

(1) For $\alpha<\beta, c^{-}(v, \alpha) \leq c^{-}(v, \beta)$ and $c^{+}(v, \alpha) \geq c^{+}(v, \beta)$.

(2) For $\alpha \leq-\|v\|, c^{-}(v, \alpha)=0$ and for $\alpha \geq\|v\|, c^{+}(v, \alpha)=0$.

(3) For $\alpha>\|v\|, c^{-}(v, \alpha)=e$ and for $\alpha<-\|v\|, c^{+}(v, \alpha)=e$.

(4) $v \in A C\left(c^{-}(v, \alpha), c^{+}(v, \alpha) ; \alpha \in \mathbb{R}\right)$.

(5) $C_{\alpha}^{-}(v) \leq \alpha c^{-}(v, \alpha)$ and $C_{\alpha}^{-\prime}(v) \geq \alpha c^{-}(v, \alpha)^{\prime} ; C_{\alpha}^{+}(v) \geq \alpha c^{+}(v, \alpha)$ and $C_{\alpha}^{+\prime}(v) \leq \alpha c^{+}(v, \alpha)^{\prime}$ for each $\alpha \in \mathbb{R}$. Here $C_{\alpha}^{ \pm}:=C_{c^{ \pm}(v, \alpha)}$.

Proof. Since $c^{+}(v, \alpha)=c^{-}(-v,-\alpha)$, we need to prove results only related to $c^{-}(v, \alpha)$.

(1). Let $\alpha<\beta$ and set $\lambda=\beta-\alpha>0$. Put $v_{\beta}=v-\beta e$ so that $c^{-}(v, \beta)$ is the cover of $v_{\beta}^{-}$. As $v_{\beta}^{-} \perp_{\infty}^{a} v_{\beta}^{+}$, we see that $v_{\beta}^{+} \perp_{\infty}^{a} c^{-}(v, \beta)$. Thus

$$
\begin{aligned}
(v-\alpha e)^{-} & =\left(v_{\beta}+\lambda e\right)^{-} \\
& =\left(v_{\beta}^{+}-v_{\beta}^{-}+\lambda c^{-}(v, \beta)+\lambda c^{-}(v, \beta)^{\prime}\right)^{-} \\
& =\left(v_{\beta}^{+}+\lambda c^{-}(v, \beta)^{\prime}\right)^{-}+\left(-v_{\beta}^{-}+\lambda c^{-}(v, \beta)\right)^{-} \\
& =\left(-v_{\beta}^{-}+\lambda c^{-}(v, \beta)\right)^{-} \in V_{c^{-}(v, \beta)}^{+}
\end{aligned}
$$

Therefore, $c^{-}(v, \alpha) \leq c^{-}(v, \beta)$, if $\alpha<\beta$.

(2). Let $\alpha \leq-\|v\|$. Then $v-\alpha e \in V^{+}$so that $(v-\alpha e)^{-}=0$. Thus $c^{-}(v, \alpha)=0$.

(3). Let $\alpha>\|v\|$. Set $k=(\alpha-\|v\|)>0$. Then

$$
(v-\alpha e)^{-}=\alpha e-v=(\|v\| e-v)+k e \geq k e .
$$

As $k>0$, we conclude that $e \in V_{c^{-}(v, \alpha)}^{+}$. Thus $e \leq c^{-}(v, \alpha)$ and consequently, $c^{-}(v, \alpha)=e$.

(4). Fix $\alpha \in \mathbb{R}$. Then

$$
v-\alpha e=(v-\alpha c(v))^{+}-(v-\alpha c(v))^{-}-\alpha c(v)^{\prime} \in V_{\bar{u}_{\alpha}^{+}}+V_{\bar{u}_{\alpha}^{-}}+V_{c(v)^{\prime}} .
$$

If $\alpha>0$, then $c^{-}(v, \alpha)=\bar{u}_{\alpha}^{-}+c(v)^{\prime}$ and $c^{+}(v, \alpha)=\bar{u}_{\alpha}^{+}$. As $\bar{u}_{\alpha}^{-}, \bar{u}_{\alpha}^{+}$and $c(v)^{\prime}$ are mutually absolutely $\infty$-orthogonal with $\bar{u}_{\alpha}^{-}+\bar{u}_{\alpha}^{+}+c(v)^{\prime} \leq e$, we get that $\bar{u}_{\alpha}^{+} \leq c^{-}(v, \alpha)^{\prime}$. Thus $V_{\bar{u}_{\alpha}^{+}}+V_{\bar{u}_{\alpha}^{-}}+V_{c(v)^{\prime}} \subset V_{c^{-}(v, \alpha)}+V_{c^{-}(v, \alpha)^{\prime}}$ whence $v-\alpha e \in$ $A C\left(c^{-}(v, \alpha)\right)$. As $e \in A C\left(c^{-}(v, \alpha)\right)$ and as the later is a subspace of $V$, we further conclude that $v \in A C\left(c^{-}(v, \alpha)\right)$. The other cases may be proved in a similar way. 
(5). Let $\alpha \in \mathbb{R}$. Then $C_{\alpha}^{-}(v-\alpha e)=-(v-\alpha e)^{-}$and $C_{\alpha}^{-}(e)=c^{-}(v, \alpha)$. Thus $C_{\alpha}^{-}(v)=-(v-\alpha e)^{-}+\alpha c^{-}(v, \alpha) \leq \alpha c^{-}(v, \alpha)$. We can prove the other statements In a similar manner.

Theorem 7.7. Let $v \in A C(p)$ for some $p \in O P(V)$. Then for any $\alpha \in \mathbb{R}$, both $c^{+}(v, \alpha)$ and $c^{-}(v, \alpha)$ are absolutely compatible with $p$.

Proof. First, let $v \in V^{+}$and $\alpha \geq 0$. As $v \in A C^{+}(p)$, we have $v=C_{p}(v)+C_{p}^{\prime}(v)$ with $C_{p}(v) \in V_{p}^{+}$and $C_{p}^{\prime} \in V_{p}^{\prime}$. Thus

$$
\begin{aligned}
(v-\alpha e)^{+} & =\left(\left(C_{p}(v)-\alpha p\right)+\left(C_{p}^{\prime}(v)-\alpha p^{\prime}\right)\right)^{+} \\
& =\left(C_{p}(v)-\alpha p\right)^{+}+\left(C_{p}^{\prime}(v)-\alpha p^{\prime}\right)^{+} .
\end{aligned}
$$

Also

$$
\begin{aligned}
\left(C_{p}(v)-\alpha e\right)^{+} & =\left(C_{p}(v)-\alpha p-\alpha p^{\prime}\right)^{+} \\
& =\left(C_{p}(v)-\alpha p\right)^{+}+\left(-\alpha p^{\prime}\right)^{+} \\
& =\left(C_{p}(v)-\alpha p\right)^{+}
\end{aligned}
$$

and

$$
\begin{aligned}
\left(C_{p}^{\prime}(v)-\alpha e\right)^{+} & =\left(C_{p}(v)-\alpha p-\alpha p^{\prime}\right)^{+} \\
& =\left(C_{p}^{\prime}(v)-\alpha p^{\prime}\right)^{+}+(-\alpha p)^{+} \\
& =\left(C_{p}^{\prime}(v)-\alpha p^{\prime}\right)^{+} .
\end{aligned}
$$

Thus $\left(C_{p}(v)-\alpha e\right)^{+} \perp_{\infty}^{a}\left(C_{p}^{\prime}(v)-\alpha e\right)^{+}$so that $c^{+}\left(C_{p}(v), \alpha\right) \perp_{\infty}^{a} c^{+}\left(C_{p}^{\prime}(v), \alpha\right)$ with $c^{+}\left(C_{p}(v), \alpha\right)+c^{+}\left(C_{p}^{\prime}(v), \alpha\right)=c^{+}(v, \alpha)$. Since $c^{+}\left(C_{p}(v), \alpha\right) \in V_{p}^{+}$and $c^{+}\left(C_{p}^{\prime}(v), \alpha\right) \in V_{p^{\prime}}^{+}$, we further see that $c^{+}\left(C_{p}(v), \alpha\right) \leq c^{+}(v, \alpha) \wedge p$ and that $c^{+}\left(C_{p}^{\prime}(v), \alpha\right) \leq c^{+}(v, \alpha) \wedge p^{\prime}$. Thus

$$
\begin{aligned}
c^{+}(v, \alpha) & =c^{+}\left(C_{p}(v), \alpha\right)+c^{+}\left(C_{p}^{\prime}(v), \alpha\right) \\
& \leq c^{+}(v, \alpha) \wedge p+c^{+}(v, \alpha) \wedge p^{\prime} \\
& \leq c^{+}(v, \alpha) .
\end{aligned}
$$

Now, it follows that $c^{+}(v, \alpha) \in A C^{+}(p)$ and that $c^{+}\left(C_{p}(v), \alpha\right)=c^{+}(v, \alpha) \wedge p$ and $c^{+}\left(C_{p}^{\prime}(v), \alpha\right)=c^{+}(v, \alpha) \wedge p^{\prime}$. Now, as $c^{+}(v, \alpha)=e$ whenever $v \in V^{+}$and $\alpha<0$, we get that $c^{+}(v, \alpha) \in A C^{+}(p)$ for any $v \in A C^{+}(p)$ and $\alpha \in \mathbb{R}$. Now, since $v-\alpha e=(v+\|v\| e)-(\alpha+\|v\|) e$ and $(v+\|v\| e) \in A C^{+}(p)$ whenever $v \in A C(p)$, we further conclude that $c^{+}(v, \alpha) \in A C^{+}(p)$ whenever $v \in A C(p)$ and $\alpha \in \mathbb{R}$. Finally, as $c^{-}(v, \alpha)=c^{+}(-v,-\alpha)$, the result holds.

Proposition 7.8. Assume that $v \in A C(p)$ for some $p \in O P(V)$ and that $\alpha \in \mathbb{R}$. Then $C_{p}(v) \leq \alpha p$ and $C_{p}^{\prime}(v) \geq \alpha p^{\prime}$ if and only if $c^{-}(v, \alpha) \leq p \leq c^{+}(v, \alpha)^{\prime}$. Also in this case, $C_{p}(v-\alpha e)=-(v-\alpha e)^{-}$and $C_{p}^{\prime}(v-\alpha e)=(v-\alpha e)^{+}$.

Proof. Find $v_{1}$ and $v_{2}$ in $V^{+}$such that $C_{p}(v)+v_{1}=\alpha p$ and $C_{p}^{\prime}(v)-v_{2}=\alpha p^{\prime}$. Then $v_{1} \in V_{p}^{+}$and $v_{2} \in V_{p^{\prime}}^{+}$so that $v_{1} \perp_{\infty}^{a} v_{2}$. Next, as $v \in A C(p)$, we have

$$
v=C_{p}(v)+C_{p^{\prime}}^{\prime}(v)=\left(\alpha p-v_{1}\right)+\left(\alpha p^{\prime}+v_{2}\right)=\alpha e+\left(v_{2}-v_{1}\right) .
$$


Thus $v-\alpha e=v_{2}-v_{1}$ and consequently, $v_{2}=(v-\alpha e)^{+}$and $v_{1}=(v-\alpha e)^{-}$for $v_{2} \perp_{\infty}^{a} v_{1}$. Now it follows that

$$
(v-\alpha e)^{+}=C_{p}^{\prime}(v)-\alpha p^{\prime}=C_{p}^{\prime}(v-\alpha e)
$$

and

$$
(v-\alpha e)^{-}=-\left(C_{p}(v)-\alpha p\right)=-C_{p}(v-\alpha e) .
$$

Further, we see that $(v-\alpha e)^{+} \in V_{p^{\prime}}^{+}$and that $(v-\alpha e)^{-} \in V_{p}^{+}$. Thus $c^{+}(v, \alpha) \leq p^{\prime}$ and $c^{-}(v, \alpha)^{-} \leq p$ so that $c^{-}(v, \alpha) \leq p \leq c^{+}(v, \alpha)^{\prime}$.

Conversely, assume that $c^{-}(v, \alpha) \leq p \leq c^{+}(v, \alpha)^{\prime}$. Then by Proposition 7.4, $C_{\alpha}^{+\prime} C_{p}=C_{p}=C_{p} C_{\alpha}^{+\prime}$ and $C_{\alpha}^{-\prime} C_{p}^{\prime}=C_{p}^{\prime}=C_{p}^{\prime} C_{\alpha}^{-\prime}$. Thus

$$
C_{p}(v-\alpha e)=C_{p} C_{\alpha}^{+\prime}(v-\alpha e) \leq 0
$$

so that $C_{p}(v) \leq \alpha p$ and

$$
C_{p}^{\prime}(v-\alpha e)=C_{p}^{\prime} C_{\alpha}^{+\prime}(v-\alpha e) \geq 0
$$

so that $C_{p}^{\prime}(v) \geq \alpha p^{\prime}$.

Proposition 7.9. Let $(V, e)$ be a monotone complete absolute order unit space in which $O P(V)$ covers $V$ and let $v \in V$. Set $e_{\alpha}=c^{+}(v, \alpha)^{\prime}$ for each $\alpha \in \mathbb{R}$. Then $\wedge_{\alpha>\alpha_{0}} e_{\alpha}=e_{\alpha_{0}}$ for each $\alpha_{0} \in \mathbb{R}$.

Proof. First note that $\wedge_{\alpha>\alpha_{0}} e_{\alpha}$ exists in $V$ as the later is monotone complete. We write $\wedge_{\alpha>\alpha_{0}} e_{\alpha}=v_{0}$ so that $v_{0} \in[0, e]$. Let $e_{0} \in O P(V)$ be the cover of $v_{0}$. Then $v_{0} \leq e_{0}$. Now, by the definition of the cover, $e_{0} \leq e_{\alpha}$ for each $\alpha>\alpha_{0}$ as $v_{0} \leq e_{\alpha}$ for such $\alpha$. Thus $e_{0} \leq v_{0}$ and we have $e_{0}=\wedge_{\alpha>\alpha_{0}} e_{\alpha} \in O P(V)$. Also, then $e_{\alpha_{0}} \leq e_{0} \leq e_{\alpha}$ if $\alpha>\alpha_{0}$. Fix $\alpha>\alpha_{0}$. Then $C_{e_{0}} C_{e_{\alpha}}=C_{e_{\alpha}} C_{e_{0}}=C_{e_{0}}$ so that

$$
C_{e_{0}}(v)=C_{e_{0}} C_{e_{\alpha}}(v) \leq C_{e_{0}}\left(\alpha e_{\alpha}\right)=\alpha e_{0} .
$$

Thus $C_{e_{0}}(v) \leq \alpha_{0} e_{0}$. Similarly, as $e_{\alpha_{0}} \leq e_{0}$, we have $e_{0}^{\prime} \leq e_{\alpha_{0}}^{\prime}$. Thus as above, we may get that $C_{e_{0}}^{\prime}(v) \geq \alpha_{0} e_{0}^{\prime}$. Now, applying Proposition 7.8, we can conclude that $e_{0}=c^{+}\left(v, \alpha_{0}\right)^{\prime}:=e_{\alpha_{0}}$.

Theorem 7.10 (Spectral Resolution). Let $(V, e)$ be a monotone complete absolute order unit space in which $O P(V)$ covers $V$ and let $v \in V$. Then there exists a unique family $\left\{e_{\alpha}: \alpha \in \mathbb{R}\right\} \subset O P(V)$ such that

(1) $\left\{e_{\alpha}: \alpha \in \mathbb{R}\right\}$ is increasing;

(2) $e_{\alpha}=0$ if $\alpha<-\|v\|$ and $e_{\alpha}=e$ if $\alpha \geq\|v\|$;

(3) $v \in O C\left(e_{\alpha} ; \alpha \in \mathbb{R}\right)$;

(4) $C_{e_{\alpha}}(v) \leq \alpha e_{\alpha}$ and $C_{e_{\alpha}}^{\prime}(v) \geq \alpha e_{\alpha}^{\prime}$ for each $\alpha \in \mathbb{R}$;

(5) If $v \in A C(p)$ for some $p \in O P(V)$ and if $C_{p}(v) \leq \alpha p$ and $C_{p}^{\prime}(v) \geq \alpha p^{\prime}$, then $p \leq e_{\alpha}$

(6) $\wedge_{\alpha>\alpha_{0}} e_{\alpha}=e_{\alpha_{0}}$ for each $\alpha_{0} \in \mathbb{R}$.

Proof. Set $e_{\alpha}=c^{+}(v, \alpha)^{\prime}$ for each $\alpha \in \mathbb{R}$. Then (1), (2) and (3) follow from Proposition 7.6; (4) and (5) follow from Proposition 7.8 and (6) follows from Proposition 7.9. Conversely, assume that a family $\left\{e_{\alpha}: \alpha \in \mathbb{R}\right\} \subset O P(V)$ satisfies conditions (1) $-(6)$. Then by Proposition 7.8, condition (4) yields $c^{-}(v, \alpha) \leq e_{\alpha} \leq$ $c^{+}(v, \alpha)^{\prime}$ and condition (5) yields $c^{+}(v, \alpha)^{\prime} \leq e_{\alpha}$ for each $\alpha \in \mathbb{R}$. This completes the proof. 
Definition 7.11. Let $(V, e)$ be a monotone complete absolute order unit space in which $O P(V)$ covers $V$ and let $v \in V$. Then an increasing family $\left\{e_{\alpha}: \alpha \in\right.$ $\mathbb{R}\} \subset O P(V)$ is called the spectral resolution of $v$ in $O P(V)$ if the family satisfies conditions (1) - (6) of Theorem 7.10.

Theorem 7.12 (Spectral Decomposition). Let $(V, e)$ be a monotone complete absolute order unit space in which $O P(V)$ covers $V$ and let $v \in V$. Consider the spectral resolution $\left\{e_{\alpha}: \alpha \in \mathbb{R}\right\}$ of $v$ in $O P(V)$. Then for any $\epsilon>0$ and a finite increasing sequence $\alpha_{0}<\cdots<\alpha_{n}$ with $\alpha_{0}<-\|v\|, \alpha_{n}>\|v\|$ and $\max \left\{\alpha_{i}-\alpha_{i-1}\right.$ : $1 \leq i \leq n\}<\epsilon$, we have

$$
\left\|v-\sum_{i=1}^{n} \alpha_{i}\left(e_{\alpha_{i}}-e_{\alpha_{i-1}}\right)\right\|<\epsilon
$$

Proof. Let $\alpha<\beta$. Then $e_{\alpha} \leq e_{\beta}$ so that by Proposition 7.5(5), we get

$$
C_{\beta}(v) \leq \beta e_{\beta}=\beta C_{\beta}(e) \text { and } C_{\alpha}^{\prime}(v) \geq \alpha e_{\alpha}^{\prime}=\alpha C_{\alpha}^{\prime}(e)
$$

As $C_{\alpha}^{\prime}$ and $C_{\beta}$ are positive operators on $O C\left(e_{\alpha}, e_{\beta}\right)$, we get

$$
\alpha C_{\beta} C_{\alpha}^{\prime}(e) \leq C_{\beta} C_{\alpha}^{\prime}(v)=C_{\alpha}^{\prime} C_{\beta}(v) \leq \beta C_{\alpha}^{\prime} C_{\beta}(e)
$$

Since $\alpha<\beta$, by Proposition 7.4, we see that

$$
C_{\beta} C_{\alpha}^{\prime}(e)=C_{\alpha}^{\prime} C_{\beta}(e)=C_{\beta}(e)-C_{\alpha}(e)=e_{\beta}-e_{\alpha}
$$

and

$$
C_{\beta} C_{\alpha}^{\prime}(v)=C_{\alpha}^{\prime} C_{\beta}(v)=C_{\beta}(v)-C_{\alpha}(v)
$$

Thus we obtain

$$
\alpha\left(e_{\beta}-e_{\alpha}\right) \leq\left(C_{\beta}(v)-C_{\alpha}(v)\right) \leq \beta\left(e_{\beta}-e_{\alpha}\right) .
$$

Applying this for $\beta=\alpha_{i}$ and $\alpha=\alpha_{i-1}$, where $1 \leq i \leq n$ and adding together, we get

$$
\sum_{i=1}^{n} \alpha_{i-1}\left(e_{\alpha_{i}}-e_{\alpha_{i-1}}\right) \leq C_{\alpha_{n}}(v)-C_{\alpha_{0}}(v) \leq \sum_{i=1}^{n} \alpha_{i}\left(e_{\alpha_{i}}-e_{\alpha_{i-1}}\right) .
$$

Now, $e_{\alpha_{n}}=e$ and $e_{\alpha_{0}}=0$ so that $C_{\alpha_{n}}(v)=v$ and $C_{\alpha_{0}}(v)=0$. Also, $\left(e_{\alpha_{i}}-\right.$ $\left.e_{\alpha_{i-1}}\right) \perp_{\infty}^{a}\left(e_{\alpha_{j}}-e_{\alpha_{j-1}}\right)$ if $i \neq j$ so that

$$
\begin{aligned}
\| \sum_{i=1}^{n} \alpha_{i}\left(e_{\alpha_{i}}-e_{\alpha_{i-1}}\right) & -\sum_{i=1}^{n} \alpha_{i-1}\left(e_{\alpha_{i}}-e_{\alpha_{i-1}}\right) \| \\
& =\left\|\sum_{i=1}^{n}\left(\alpha_{i}-\alpha_{i-1}\right)\left(e_{\alpha_{i}}-e_{\alpha_{i-1}}\right)\right\| \\
& =\max \left\{\alpha_{i}-\alpha_{i-1}: 1 \leq i \leq n\right\}<\epsilon .
\end{aligned}
$$

Thus

$$
\left\|v-\sum_{i=1}^{n} \alpha_{i}\left(e_{\alpha_{i}}-e_{\alpha_{i-1}}\right)\right\|<\epsilon
$$




\section{REFERENCES}

1. M. C. Abbati and A. Mania, A spectral theory for order unit spaces, Ann. Inst. H. Poincare Sect. A (N.S.) 35 (1981), 259-285.

2. E. M. Alfsen and F. W. Shultz, Geometry of state spaces of operator algebras, Birkhauser, Berlin, 2002.

3. L. Asimow, Universally well-capped cones, Pacific J. Math., 26(3) (1968), 421- 431.

4. P. Bonnet, Une theorie spectrale dans certains espaces du type A(K), C. R. Acad. SCL Pans Ser. A-B 28 (1976), A207-A210.

5. M. D. Choi and E. G. Effros, Injectivity and operator spaces, J. Funct. Anal., 24 (1977), 156- 209.

6. R. V. Kadison, A representation theory for commutative topological algebras, Mem. Amer. Math. Soc., 7 (1951).

7. S. Kakutani, Concrete representation of abstract (M)- spaces, Ann. Math. 42(4) (1941), 994- 1024.

8. A. K. Karn, Order units in a $C^{*}$-algebra, Proc. of Indian Academy of Sciences, 14 (2002), 441- 458.

9. A. K. Karn, A p-theory of ordered normed spaces, Positivity 14 (2010), 441- 458.

10. A. K. Karn, Order embedding of a matrix ordered space, Bulletin Aust. Math. Soc., 84 (2011), 10- 18.

11. A. K. Karn, Orthogonality in $\ell_{p}$-spaces and its bearing on ordered normed spaces, Positivity 18 (2014), 223- 234.

12. A. K. Karn, Orthogonality in $C^{*}$-algebras, Positivity 20(3) (2016), 607- 620.

13. A. K. Karn and R. Vasudevan, Approximate matrix order unit spaces, Yokohama Math. J., 44 (1997) 73- 91.

14. K. F. Ng, The duality of partially ordered Banach spaces, Bull. Lond. Math. Soc, 19 (1969), 269- 288.

School of Mathematical Science, National Institute of Science Education and

Research, HBNi, Bhubaneswar, At \& Post - Jatni, PIN - 752050, India.

E-mail address: anilkarn@niser.ac.in; anil.karn@gmail.com 